\title{
Bain: A program for Bayesian testing of order constrained hypotheses in structural equation models
}

Xin $\mathrm{Gu}^{1}$, Herbert Hoijtink ${ }^{2}$, Joris Mulder ${ }^{3}$, and Yves Rosseel ${ }^{4}$

${ }^{1}$ Department of Educational Psychology, East China Normal University, China

${ }^{2}$ Department of Methodology and Statistics, Utrecht University, The Netherlands; CITO Institute for Educational Measurement, Arnhem, The Netherlands

${ }^{3}$ Department of Methodology and Statistics, Tilburg University, The Netherlands

${ }^{4}$ Department of Data Analysis, Ghent University, Belgium

October 19, 2018

"This is an Accepted Manuscript of an article published by Taylor \& Francis in the Journal of Statistical Computation and Simulation 2019, available online: http://www.tandfonline.com/ with DOI https://doi.org/10.1080/00949655.2019.1590574 ."

\footnotetext{
${ }^{1}$ Corresponding address: guxin57@hotmail.com (Xin Gu)

${ }^{2}$ Herbert Hoijtink is supported by the Consortium on Individual Development (CID) which is funded through the Gravitation program of the Dutch Ministry of Education, Culture, and Science and the Netherlands Organization for Scientific Research (NWO grant number 024.001.003).

${ }^{3}$ Joris Mulder was supported by a Vidi Grant of the Netherlands Organization for Scientific Research (NWO grant number 452-17-006).
} 


\begin{abstract}
This paper presents a new statistical method and accompanying software for the evaluation of order constrained hypotheses in structural equation models (SEM). The method is based on a large sample approximation of the Bayes factor using a prior with a data-based correlational structure. An efficient algorithm is written into an $\mathrm{R}$ package to ensure fast computation. The package, referred to as Bain, is easy to use for applied researchers. Two classical examples from the SEM literature are used to illustrate the methodology and software.

Keywords: Approximate Bayesian procedure, Bayes factors, Order constrained hypothesis, Structural equation model.
\end{abstract}

\title{
1 Introduction
}

Applied researchers have become increasingly interested in the evaluation of order constrained hypotheses because the traditional null hypothesis is often not a realistic representation of the population of interest (Cohen, 1994; Royall 1997, pp.79-81). In structural equation models, researchers may have explicit theories or expectations, for example, about the ordering of the relative effects of independent variables on a dependent variable or researchers may expect which indicator for a latent variable is dominant over the other indicators. These expectations can be represented by order constrained hypotheses among the model parameters. Order constrained hypotheses can be evaluated using either the frequentist approach by means of $p$ values (see, e.g., Silvapulle and Sen, 2005; van de Schoot, Hoijtink, and Deković, 2010) or the Bayesian approach by means of Bayes factors (see, e.g., van de Schoot, Hoijtink, Hallquist, and Boelen, 2012; Klugkist, Laudy, and Hoijtink, 2005; Hoijtink, 2012). In this paper, the Bayes factor (Kass and Raftery, 1995) is used as a criterion for assessing the hypotheses because $p$ values can only reject a null hypothesis. Bayes factors on the other hand are able to measure the relative evidence in the data between multiple nonnested hypotheses containing order constraints (Wagenmakers, 2007). For this reason, Bayes factors can be viewed as a more generally applicable tool for statistical hypothesis testing than classical $p$ values.

During the past decade, Bayesian evaluation of hypotheses with order (or inequality) constraints on the parameters of interest has been studied for various statistical models. Besides statistical theory development, these studies rendered software packages that can be used by applied researchers, see Hoijtink (2012, pp.179) for an overview. As a pioneer, Klugkist, Laudy, and Hoijtink (2005) presented a Bayesian approach to evalu- 
ate analysis of (co)variance models (ANOVA or ANCOVA) with order constraints on the means. The study for ANOVA models was further developed by Kuiper and Hoijtink (2010) for the comparison of means using both Bayesian and non-Bayesian methods. This research resulted in a software package Conf irmatoryANOVA (Kuiper, Klugkist, and Hoijtink, 2010). Thereafter, Mulder, Hoijtink, and Klugkist (2010) extended the previous study to multivariate linear models (MANOVA, repeated measures, multivariate regression), which is implemented in the software package BIEMS (Mulder, Hoijtink and, de Leeuw, 2012). Finally, Gu, Mulder, Decović, and Hoijtink (2014) explored a general Bayesian procedure using a diffuse normal prior distribution with a diagonal covariance structure. Although this methodology provided reasonable default outcomes of the Bayes factor, the diagonal prior covariance structure can be criticized because the resulting Bayes factor is not invariant for linear one-to-one transformations of the data. The invariance property is important because it ensures that the relative evidence between two hypotheses, as quantified by the Bayes factor, does not depend on the arbitrary parameterizations of the model (Mulder, 2014a).

To illustrate the issue, consider three repeated measurements coming from a multivariate normal distribution, i.e., $\mathbf{x}_{i}=\left(x_{i 1}, x_{i 2}, x_{i 3}\right)^{T} \sim N(\boldsymbol{\theta}, \boldsymbol{\Sigma})$, where $\boldsymbol{\theta}=\left(\theta_{1}, \theta_{2}, \theta_{3}\right)^{T}$ is a vector containing the measurement means and $\boldsymbol{\Sigma}$ is the measurement covariance matrix. Now assume we are interested in testing a monotonic increase of the means against an unrestricted alternative: $H_{1}: \theta_{1}<\theta_{2}<\theta_{3}$ versus $H_{u}: \boldsymbol{\theta} \in \mathbb{R}^{3}$ where $\mathbb{R}^{3}$ denotes the 3 -dimensional real vector space. A standard choice for the prior under $H_{1}$ is to use a truncation of the unconstrained prior under $H_{u}$ in the order constrained space under $H_{1}$ (e.g., Klugkist et al., 2005). This results in the following expression of the Bayes factor:

$$
B_{1 u}=\frac{\operatorname{Pr}\left(\theta_{1}<\theta_{2}<\theta_{3} \mid \mathbf{X}, H_{u}\right)}{\operatorname{Pr}\left(\theta_{1}<\theta_{2}<\theta_{3} \mid H_{u}\right)},
$$

which corresponds to the ratio of the posterior probability that the constraints of $H_{1}$ are satisfied under $H_{u}$ and the prior probability that the constraints of $H_{1}$ are satisfied under $H_{u}$. Now consider a very vague prior with a multivariate normal distribution for the measurement means under $H_{u}$ with a diagonal covariance structure, $\pi_{u}(\boldsymbol{\theta})=N\left(\mathbf{0}, \omega \mathbf{I}_{3}\right)$, where $\mathbf{I}_{3}$ is a 3 -dimensional identity matrix and $\omega$ is chosen large enough so that the posterior probability in the numerator in (1) is virtually independent of the prior, say, $\omega=10^{6}$. This prior results in a prior probability that the constraints hold that is equal to $\frac{1}{6}$ (for any choice of $\omega$, see Klugkist, Laudy, and Hoijtink, 2005), and thus, the Bayes factor is equal to the pos- 
terior probability that the measurement means increase multiplied by 6 , i.e., $B_{1 u}=6 \times \operatorname{Pr}\left(\theta_{1}<\theta_{2}<\theta_{3} \mid \mathbf{X}, H_{u}\right)$.

Now we consider a one-to-one transformation of the data where the first element corresponds to the difference between the first and second repeated measurement, the second element corresponds to the difference between the second and third repeated measurement, and the third element corresponds to the third repeated measurement, i.e., $\mathbf{y}_{i}=\left(y_{i 1}, y_{i 2}, y_{i 3}\right)=\left(x_{i 1}-x_{i 2}, x_{i 2}-\right.$ $\left.x_{i 3}, x_{i 3}\right)$. Again the transformed observations follow a multivariate normal distribution, say, $\mathbf{y}_{i} \sim N(\boldsymbol{\eta}, \Psi)$, where the first, second, and third element of $\boldsymbol{\eta}$ are equal to the first mean difference, the second mean difference, and the mean of the third observation. The equivalent hypothesis test in this parameterization comes down to $H_{1}: \eta_{1}<0, \eta_{2}<0, \eta_{3} \in \mathbb{R}^{1}$ versus $H_{u}$ : $\boldsymbol{\eta} \in \mathbb{R}^{3}$. Similarly as in (1), the Bayes factor is now given by

$$
B_{1 u}=\frac{\operatorname{Pr}\left(\eta_{1}<0, \eta_{2}<0 \mid \mathbf{Y}, H_{u}\right)}{\operatorname{Pr}\left(\eta_{1}<0, \eta_{2}<0 \mid H_{u}\right)}
$$

Again we consider independent normal priors for the mean parameters, i.e., $\pi_{u}(\boldsymbol{\eta})=N\left(\mathbf{0}, \omega \mathbf{I}_{3}\right)$, with $\omega$ very large. In this case the prior probability that the constraints of $H_{1}$ hold under $H_{u}$ equals $\frac{1}{4}$, and consequently, the Bayes factor is equal to the posterior probability of negative mean differences (which is equivalent to an increase of the measurement means) multiplied by 4, i.e., $B_{1 u}=4 \times \operatorname{Pr}\left(\eta_{1}<0, \eta_{2}<0 \mid \mathbf{Y}, H_{u}\right)$. Thus, the Bayes factor differs with a factor of $\frac{4}{6}$ for these two parameterizations, which is quite large. For larger dimensions with, say, 10 measurements, the violation will be even larger (Mulder, 2014a; 2014b). This is highly undesirable. To resolve this we present a new default prior resulting in a new Bayesian testing procedure for testing order constrained hypotheses in SEM which avoids this issue. The general idea is to let the prior covariance structure of the parameters of interest to depend on the covariance structure in the sample.

The second main contribution is the development of an efficient algorithm for computing the prior and posterior probability that a set of order (inequality) constraints hold, which are key quantities when computing Bayes factors. This contribution is needed because computing these probabilities as the proportion of draws satisfying the constraints can be very inefficient when the hypotheses contain many order constraints on the parameters of interest. In this case the posterior and prior probability that the constraints hold can be very small and therefore billions of draws may be needed in order to get accurate estimates of the probabilities and the resulting Bayes factors (Hoijtink, 2012). For this reason an efficient algorithm is presented that consists of roughly two steps. First, the probability of a set of order constraints 
is written as product of conditional probabilities. Second, the conditional probabilities are computed as the arithmetic mean of conditional probabilities which have analytic expressions in the Gibbs sampler. As will be seen this new algorithm is much more efficient than the use of the proportion of draws satisfying the constraints.

The algorithm is implemented into an $\mathrm{R}$ package referred to as Bain to ensure fast computation. For the computation of the Bayes factor using Bain the user only needs to provide the estimates of the parameters of interest and the inverse of the Fisher information matrix of the parameters which serves as the posterior covariance matrix. These statistics can for instance be obtained using the lavaan package (Rosseel, 2012) in $\mathrm{R}$ for the analysis of structural equation models (SEM). Other software, such as Mplus, can also be used to obtain these statistics, but here we will use lavaan as the basis for our analyses because it is free.

In what follows, Section 2 shortly introduces SEM models and defines order constrained hypotheses. For the evaluation of order constrained hypotheses, the Bayes factor as a criterion is briefly introduced in Section 3. Subsequently, Section 4 specifies prior and posterior distributions which are the determinants of the Bayes factor. Thereafter, the procedure for the computation of Bayes factors is presented in Section 5 in which seven subsections describe the principles and algorithms used. To illustrate how to evaluate order constrained hypotheses using our program, Section 6 analyzes two classic SEM models: confirmatory factor analysis and multiple regression models with latent variables. Finally, a user manual is provided in Appendix B such that researchers can use the implementation in Bain successfully for the analysis of their own data.

\section{Order constrained structural equation models}

\subsection{Structural equation models}

The structural equation model (SEM) mainly consists of two components, i.e., the measurement model which expresses the relations between latent variables and their indicators, and the structural model which expresses the relations between endogenous and exogenous (latent) variables, see for example Jöreskog and Sörbom (1979). The measurement model can be written by

$$
\begin{aligned}
& \boldsymbol{y}=\boldsymbol{\Lambda}_{y} \boldsymbol{\eta}+\boldsymbol{\epsilon}_{y} \\
& \boldsymbol{x}=\boldsymbol{\Lambda}_{x} \boldsymbol{\xi}+\boldsymbol{\epsilon}_{x}
\end{aligned}
$$


where $\boldsymbol{y}$ and $\boldsymbol{x}$ denote the vectors of endogenous and exogenous observed variables, respectively, $\boldsymbol{\eta}$ and $\boldsymbol{\xi}$ denote the vectors of endogenous and exogenous latent variables, respectively, $\boldsymbol{\Lambda}_{y}$ and $\boldsymbol{\Lambda}_{x}$ are the corresponding matrices of factor loadings, and the measurement errors $\boldsymbol{\epsilon}_{y}$ and $\boldsymbol{\epsilon}_{x}$ have zero means and covariance matrices $\boldsymbol{\Psi}_{\epsilon_{y}}$ and $\boldsymbol{\Psi}_{\epsilon_{x}}$, respectively.

The structural model represents the relations among latent variables:

$$
\eta=\mathbf{B} \eta+\Gamma \xi+\delta,
$$

where $\mathbf{B}$ and $\boldsymbol{\Gamma}$ are matrices of regression coefficients, and $\boldsymbol{\delta}$ with mean of $\mathbf{0}$ and covariance matrix of $\boldsymbol{\Psi}_{\delta}$ is the error term. In addition,

$$
\boldsymbol{\Phi}_{\eta}=(\mathbf{I}-\mathbf{B})^{-1}\left(\boldsymbol{\Gamma} \boldsymbol{\Phi}_{\xi} \boldsymbol{\Gamma}^{T}+\mathbf{\Psi}_{\delta}\right)\left(\mathbf{I}^{T}-\mathbf{B}^{T}\right)^{-1},
$$

where $\boldsymbol{\Phi}_{\eta}$ and $\boldsymbol{\Phi}_{\xi}$ are the covariance matrices of the latent variables $\boldsymbol{\eta}$ and $\boldsymbol{\xi}$, respectively. Note that both $\boldsymbol{\eta}$ and $\boldsymbol{\xi}$ may contain observed variables if one wants to model the relationship between observed variables. This can be done by creating single-indicator latent variables (with a fixed factor loading of 1 , and zero measurement error) corresponding to each observed variable.

The general framework of SEM is described by equations (2) and (3) which can be specified using lavaan syntax (Rosseel, 2012) in R. As can be seen from (2), (3) and (4), the non-fixed elements in $\left\{\boldsymbol{\Lambda}_{y}, \boldsymbol{\Lambda}_{x}, \mathbf{B}, \boldsymbol{\Gamma}, \boldsymbol{\Psi}_{\epsilon_{y}}, \boldsymbol{\Psi}_{\epsilon_{x}}, \boldsymbol{\Psi}_{\delta}, \boldsymbol{\Phi}_{\xi}\right\}$ of a specific SEM model can be collected in a parameter vector $\boldsymbol{\lambda}$. The density of the data is given by $f(\boldsymbol{X} \mid \boldsymbol{\lambda})$, where $\boldsymbol{X}$ denotes the data (Bollen, 1989). The distribution of data $\boldsymbol{X}$ is most often multivariate normal, though it could also involve multinomial distribution, et al. Furthermore, the nonfixed parameters can be divided into $\boldsymbol{\lambda}=\{\boldsymbol{\theta}, \boldsymbol{\zeta}\}$, where $\boldsymbol{\theta}$ denotes the target parameters that will appear in the order constrained hypotheses elaborated in the next section, and $\boldsymbol{\zeta}$ denotes the nuisance parameters that will not.

\subsection{Order constrained hypotheses}

Order constrained hypotheses express the expectations of researchers among the (standardized) target parameters in SEM. For example, hypothesis $H_{1}$ : $\theta_{1}>\theta_{2}$ where $\theta_{1}$ and $\theta_{2}$ are the coefficients of the predictors $\xi_{1}$ and $\xi_{2}$, respectively, implies that the predictor $\xi_{1}$ is stronger than $\xi_{2}$. The general form of an order constrained hypothesis $H_{i}$ is given by

$$
H_{i}: \boldsymbol{R}_{i} \boldsymbol{\theta}>\boldsymbol{r}_{i}
$$

where $\boldsymbol{R}_{i}$ is the restriction matrix containing order constraints, and $\boldsymbol{\theta}$ and $\boldsymbol{r}_{i}$ denote the target parameter vector and constant vector in $H_{i}$, respectively. 
We assume that the number of constraints is $K$ and the number of target parameters is $J$. Therefore, $\boldsymbol{R}_{i}$ is a $K \times J$ matrix, and the lengths of $\boldsymbol{\theta}$ and $\boldsymbol{r}_{i}$ are $J$ and $K$, respectively. For instance, $H_{2}: \theta_{1}>\theta_{2}>\theta_{3}$ is an example with $J=3$ and $K=2$, which leads to $\boldsymbol{\theta}=\left(\theta_{1}, \theta_{2}, \theta_{3}\right)^{\mathrm{T}}$ and an augmented matrix:

$$
\left[\boldsymbol{R}_{2} \mid \boldsymbol{r}_{2}\right]=\left[\begin{array}{ccc:c}
1 & -1 & 0 & 0 \\
0 & 1 & -1 & 0
\end{array}\right]
$$

The augmented matrix $\left[\boldsymbol{R}_{i} \mid \boldsymbol{r}_{i}\right]$ should be implemented as input of Bain.

The hypothesis $H_{i}$ is often compared to an unconstrained hypothesis

$$
H_{u}: \boldsymbol{\theta} \in \mathbb{R}^{J},
$$

where $\mathbb{R}^{J}$ denotes the $J$-dimensional real vector space, or to its complement

$$
H_{i_{c}}: \operatorname{not} H_{i} .
$$

Furthermore, we can evaluate $H_{i}$ against a competing hypothesis

$$
H_{i^{\prime}}: \boldsymbol{R}_{i^{\prime}} \boldsymbol{\theta}>\boldsymbol{r}_{i^{\prime}}
$$

The evaluation of these hypotheses can be conducted using Bayes factors, which will be elaborated in the next section.

When specifying order constrained hypotheses in SEM models, the target parameters may need to be standardized. For example, if hypothesis $H_{1}$ :

$\theta_{1}>\theta_{2}$ compares two regression coefficients to determine which predictor is stronger, then the coefficients $\theta_{1}$ and $\theta_{2}$ should be standardized to be comparable. The standardization of target parameters can be achieved by standardizing the observed and latent variables in SEM models. However, this manner might be criticized because the data is used twice, once for standardization and once for evaluation of the hypothesis ( $\mathrm{Gu}$ et al., 2014). The lavaan package (Rosseel, 2012) provides an alternative approach that can directly obtain estimates and covariance matrix of standardized target parameters. This paper uses the alternative standardization approach in lavaan. To keep the notation simple, in this paper $\boldsymbol{\theta}$ will be used to denote both unstandardized and standardized target parameters.

\section{Bayes factor}

The Bayes factor of $H_{i}$ against $H_{u}$ is defined as the ratio of two marginal likelihoods (Jeffreys, 1961; Kass and Raftery, 1995; Hoijtink, 2012):

$$
B F_{i u}=\frac{m_{i}(\boldsymbol{X})}{m_{u}(\boldsymbol{X})}=\frac{\iint f(\boldsymbol{X} \mid \boldsymbol{\theta}, \boldsymbol{\zeta}) \pi_{i}(\boldsymbol{\theta}, \boldsymbol{\zeta}) d \boldsymbol{\theta} d \boldsymbol{\zeta}}{\iint f(\boldsymbol{X} \mid \boldsymbol{\theta}, \boldsymbol{\zeta}) \pi_{u}(\boldsymbol{\theta}, \boldsymbol{\zeta}) d \boldsymbol{\theta} d \boldsymbol{\zeta}},
$$


where $\pi_{i}(\boldsymbol{\theta}, \boldsymbol{\zeta})$ and $\pi_{u}(\boldsymbol{\theta}, \boldsymbol{\zeta})$ denote the prior distribution under $H_{i}$ and $H_{u}$ (will be specified in the next section), respectively, and $f(\boldsymbol{X} \mid \boldsymbol{\theta}, \boldsymbol{\zeta})$ denotes the density of $\boldsymbol{X}$ given $\boldsymbol{\theta}$ and $\boldsymbol{\zeta}$ (see Bollen, 1989). Furthermore, from equation (9) it follows that the Bayes factor of $H_{i}$ against $H_{i_{c}}$ can be obtained as $B F_{i i_{c}}=B F_{i u} / B F_{i_{c} u}$, and the Bayes factor of $H_{i}$ against $H_{i^{\prime}}$ is $B F_{i i^{\prime}}=$ $B F_{i u} / B F_{i^{\prime} u}$.

The Bayes factor $B F_{i u}$ quantifies the relative evidence in the data in favor of hypothesis $H_{i}$ against $H_{u}$. For example $B F_{i u}=2$ indicates that the support in the data for $H_{i}$ is twice as large as the support for $H_{u}$. A general guideline for the interpretation of the Bayes factor is that $B F_{i u} \in(1,3]$ indicates evidence for $H_{i}$ that is not worth mentioning, and $B F_{i u} \in(3,20]$, $B F_{i u} \in(20,150]$ and $B F_{i u}>150$ indicate positive, strong and very strong evidence for $H_{i}$, respectively (Kass and Raftery, 1995). Note that if $B F_{i u}<1$ which implies evidence against $H_{i}$, the strength of this evidence is quantified using the rule above for the reciprocal of $B F_{i u}$. Furthermore, Bayes factors $B F_{i i_{c}}$ and $B F_{i i^{\prime}}$ can also be interpreted using the same rule. Although this rule renders a proposal to interpret the Bayes factor, it is not suggested using it strictly because this interpretation is a rough descriptive statement with respect to the standards of evidence, which could very well be modified based on the research context. For this reason users can judge by themselves when the evidence in the data is positive, strong or decisive in favor or against a hypothesis based on the observed Bayes factor.

Formula (9) can be simplified to (Klugkist and Hoijtink, 2007):

$$
B F_{i u}=\frac{f_{i}}{c_{i}}
$$

where

$$
c_{i}=\iint_{\theta \in \Theta_{i}} \pi_{u}(\boldsymbol{\theta}, \boldsymbol{\zeta}) d \boldsymbol{\theta} d \boldsymbol{\zeta}=\int_{\theta \in \Theta_{i}} \pi_{u}(\boldsymbol{\theta}) d \boldsymbol{\theta},
$$

called relative complexity (Mulder 2014b), is the proportion of the prior distribution (specified in the next section) in agreement with $H_{i}$ relative to $H_{u}$, and

$$
f_{i}=\iint_{\theta \in \Theta_{i}} \pi_{u}(\boldsymbol{\theta}, \boldsymbol{\zeta} \mid \boldsymbol{X}) d \boldsymbol{\theta} d \boldsymbol{\zeta}=\int_{\theta \in \Theta_{i}} \pi_{u}(\boldsymbol{\theta} \mid \boldsymbol{X}) d \boldsymbol{\theta}
$$

called relative fit, is the proportion of the posterior distribution (specified in the next section) in agreement with $H_{i}$ relative to $H_{u}$. Here $\Theta_{i}=\left\{\boldsymbol{\theta} \mid \boldsymbol{R}_{i} \boldsymbol{\theta}>\right.$ $\left.\boldsymbol{r}_{i}\right\}$ denotes the parameter space constrained by $H_{i}$, and $\boldsymbol{\zeta}$ is not constrained. The complexity implies how specific a hypothesis is, and the fit implies how much the data supports a hypothesis relative to $H_{u}$. The more specific the 
hypothesis, the less the complexity, while the more the support from the data, the larger the fit. The derivation of equation (10) can be found in Mulder (2014b). Equation (10) shows that the Bayes factor of an order constrained hypothesis $H_{i}$ against an unconstrained hypothesis $H_{u}$ can be represented as the ratio of the fit and complexity of $H_{i}$. This representation facilitates our development of the software for the evaluation of order constrained hypotheses.

Based on $B F_{i u}$, the Bayes factor $B F_{i i_{c}}$ for $H_{i}$ against $H_{i_{c}}$, and $B F_{i i^{\prime}}$ for two competing hypotheses $H_{i}$ and $H_{i^{\prime}}$ can also be derived. Noting that the proportions of prior and posterior distributions in agreement with $H_{i_{c}}$ are $1-c_{i}$ and $1-f_{i}$, respectively, it follows that

$$
B F_{i i_{c}}=\frac{f_{i}}{c_{i}} / \frac{1-f_{i}}{1-c_{i}} .
$$

Analogously, $B F_{i i^{\prime}}$ can be obtained by

$$
B F_{i i^{\prime}}=B F_{i u} / B F_{i^{\prime} u}=\frac{f_{i}}{c_{i}} / \frac{f_{i^{\prime}}}{c_{i^{\prime}}} .
$$

Furthermore, an accessible manner for comparing a set of hypotheses is to transform Bayes factors into posterior model probabilities (PMPs). The PMPs are a representation of the support in the data for each hypothesis on a scale between 0 and 1 . Assuming equal prior probabilities for the hypotheses, we obtain PMPs for all the competing hypotheses excluding $H_{u}$ using (Hoijtink, 2012, pp.52)

$$
P M P_{i}=\frac{B F_{i u}}{\sum_{i} B F_{i u}} \text { for } i=1, \ldots, I_{N},
$$

where $I_{N}$ denotes the number of competing hypotheses. The execution of our program renders both Bayes factors (10) and PMPs (15). As was shown in (10), the Bayes factor for $H_{i}$ against $H_{u}$ depends on the complexity and fit for which the prior and posterior distributions of $\boldsymbol{\theta}$ under $H_{u}$ need to be specified, respectively. The specification of prior and posterior distributions will be introduced in the next section.

\section{Prior and posterior distributions}

\subsection{Prior specification}

The specification of prior distributions is an important step in Bayesian hypothesis testing. As can be seen from equation (11), only a proper prior of $\boldsymbol{\theta}$ 
for the unconstrained hypothesis needs to be specified; the priors under the order constrained hypotheses automatically follow from this prior by truncating the unconstrained prior in the respective order constrained subspaces. The unconstrained prior that is proposed in this paper is partly based on the fractional Bayes factor of O'Hagan (1995) which is known to be invariant for linear transformations. In the fractional Bayes factor a prior is implicitly constructed using a small fraction of the information in the data. A key property of the resulting automatic prior is that it has the same covariance structure as the covariance structure in the data (Mulder, 2014b).

In SEM the covariance structure in the data of the parameters of interest is contained in the estimated covariance matrix of the (standardized) target parameters, denoted by $\hat{\boldsymbol{\Sigma}}_{\theta}$. This covariance matrix can be obtained by standard SEM software packages such as lavaan (Rosseel, 2012). Following the idea of a data-based covariance structure, as in the fractional Bayes factor, the following unconstrained normal prior will be used for the target parameters

$$
\pi_{u}^{*}(\boldsymbol{\theta})=N\left(\mathbf{0}, \omega \hat{\mathbf{\Sigma}}_{\theta}\right),
$$

where $\omega$ controls the amount of prior information (a small/large value for $\omega$ implies an informative/vague prior). To avoid the dependence of the (arbitrarily chosen) mean vector $\mathbf{0}$, we let $\omega$ go to $\infty$. Although extremely vague priors are not recommended when testing hypotheses with equality constraints due to Lindley-Bartlett's paradox (Lindley, 1957; Bartlett, 1957), such priors can be used for testing order constrained hypotheses (Klugkist et al., 2005).

To illustrate that the prior probability that the constraints hold is invariant for linear transformations, let us consider the following order constrained hypothesis $H_{2}: \theta_{1}>\theta_{2}>\theta_{3}$ with restriction matrix

$$
\boldsymbol{R}_{2}=\left[\begin{array}{ccc}
1 & -1 & 0 \\
0 & 1 & -1
\end{array}\right]
$$

for the repeated measures data $\boldsymbol{y}_{i}=\left(y_{i 1}, y_{i 2}, y_{i 3}\right)^{T} \sim N\left(\boldsymbol{\theta}, \boldsymbol{\Sigma}_{y}\right)$, where $\boldsymbol{\theta}=$ $\left(\theta_{1}, \theta_{2}, \theta_{3}\right)^{T}$ is a mean vector and $\boldsymbol{\Sigma}_{y}$ is a covariance matrix. Now let us consider a data set where the three measurements are independent, e.g., $\hat{\mathbf{\Sigma}}_{\theta}=\mathbf{I}_{3}$, resulting in an unconstrained prior of the form $N\left(\mathbf{0}, \omega \mathbf{I}_{3}\right)$. In this case the prior probability, which reflects the relative complexity of $H_{2}$ relative to $H_{u}$, is equal to $\operatorname{Pr}\left(\theta_{1}>\theta_{2}>\theta_{3} \mid H_{u}\right)=\frac{1}{6}$, for any choice of $\omega>0$.

Now we consider a one-to-one transformation of the data according to 
$\mathbf{z}_{i}=\left(y_{i 1}-y_{i 2}, y_{i 2}-y_{i 3}, y_{i 3}\right)^{T}=\mathbf{L} \mathbf{y}_{i}$, with

$$
\mathbf{L}=\left[\begin{array}{ccc}
1 & -1 & 0 \\
0 & 1 & -1 \\
0 & 0 & 1
\end{array}\right]
$$

We shall write $\mathbf{z}_{i} \sim N\left(\boldsymbol{\gamma}, \boldsymbol{\Sigma}_{z}\right)$, where $\boldsymbol{\gamma}=\mathbf{L} \boldsymbol{\theta}=\left(\theta_{1}-\theta_{2}, \theta_{2}-\theta_{3}, \theta_{3}\right)^{T}$ and $\boldsymbol{\Sigma}_{z}=\mathbf{L} \boldsymbol{\Sigma}_{y} \mathbf{L}^{T}$. Thus, the equivalent constrained hypothesis in the new parameterization corresponds to $H_{2}: \gamma_{1}>0, \gamma_{2}>0$. Consequently, the estimated covariance matrix is now

$$
\hat{\mathbf{\Sigma}}_{\gamma}=\mathbf{L} \hat{\mathbf{\Sigma}}_{\theta} \mathbf{L}^{T}=\mathbf{L} \mathbf{L}^{T}=\left[\begin{array}{ccc}
2 & -1 & 0 \\
-1 & 2 & -1 \\
0 & -1 & 1
\end{array}\right]
$$

This results in an unconstrained prior for the target parameters of $\left(\gamma_{1}, \gamma_{2}\right)^{T} \sim$ $N\left(\mathbf{0}, \omega\left[\begin{array}{cc}2 & -1 \\ -1 & 2\end{array}\right]\right)$. The prior probability now remains unchanged because $\operatorname{Pr}\left(\gamma_{1}>0, \gamma_{2}>0 \mid H_{u}\right)=\frac{1}{6}$, for any choice of $\omega>0$.

Theorem 1 provides a general proof of this invariance of the prior probability that the constraints of $H_{i}$ hold with respect to the mean parameters in multivariate data.

Theorem 1: The complexity of $H_{i}: \boldsymbol{R}_{i} \boldsymbol{\theta}>\boldsymbol{r}_{i}$ when using $\pi_{u}^{*}(\boldsymbol{\theta})$ is invariant for linear one-to-one transformation of the multivariate data $\boldsymbol{y} \sim N\left(\boldsymbol{\theta}, \boldsymbol{\Sigma}_{y}\right)$.

Proof: For the multivariate data, the covariance matrix of $\boldsymbol{\theta}$ is approximated by $\hat{\boldsymbol{\Sigma}}_{\theta}=\boldsymbol{S}_{Y} / n$, where $\boldsymbol{S}_{Y}=\left(\boldsymbol{Y}-\mathbf{1} \overline{\boldsymbol{y}}^{T}\right)^{T}\left(\boldsymbol{Y}-\mathbf{1} \overline{\boldsymbol{y}}^{T}\right)$ with $\overline{\boldsymbol{y}}$ being the sample means of $\boldsymbol{Y}=\left(\boldsymbol{y}_{1}, \cdots, \boldsymbol{y}_{n}\right)$. Following (16) the prior distribution for $\boldsymbol{\theta}$ is $\pi_{u}^{*}(\boldsymbol{\theta})=N\left(0, \frac{\omega}{n} \boldsymbol{S}_{Y}\right)$.

Consider a linear one-to-one transformation $\boldsymbol{L} \boldsymbol{y}=\boldsymbol{z} \sim N\left(\boldsymbol{\gamma}, \boldsymbol{\Sigma}_{z}\right)$, where $\boldsymbol{L}$ is a $J \times J$ full rank matrix, and $\boldsymbol{\gamma}=\boldsymbol{L} \boldsymbol{\theta}$ and $\boldsymbol{\Sigma}_{z}=\boldsymbol{L} \boldsymbol{\Sigma}_{y} \boldsymbol{L}^{T}$. After linear transformation, similarly, the covariance matrix of $\gamma$ is approximated by $\hat{\boldsymbol{\Sigma}}_{\gamma}=\boldsymbol{S}_{Z} / n$, where $\boldsymbol{S}_{Z}=\left(\boldsymbol{Z}-\mathbf{1} \overline{\boldsymbol{z}}^{T}\right)^{T}\left(\boldsymbol{Z}-\mathbf{1} \overline{\boldsymbol{z}}^{T}\right)$ with $\overline{\boldsymbol{z}}$ being the sample means of $\boldsymbol{Z}=\left(\boldsymbol{z}_{1}, \cdots, \boldsymbol{z}_{n}\right)$. Note that $\boldsymbol{S}_{Z}=\boldsymbol{L}\left(\boldsymbol{Y}-\mathbf{1} \overline{\boldsymbol{y}}^{T}\right)^{T}(\boldsymbol{Y}-$ $\left.\mathbf{1} \overline{\boldsymbol{y}}^{T}\right) \boldsymbol{L}^{T}=\boldsymbol{L} \boldsymbol{S}_{Y} \boldsymbol{L}^{T}$ which implies $\hat{\boldsymbol{\Sigma}}_{\gamma}=\boldsymbol{L} \hat{\boldsymbol{\Sigma}}_{\theta} \boldsymbol{L}^{T}$, then the prior distribution for $\boldsymbol{\gamma}$ becomes $\pi_{u}^{*}(\boldsymbol{\gamma})=N\left(0, \frac{\omega}{n} \boldsymbol{L} \boldsymbol{S}_{Y} \boldsymbol{L}^{T}\right)$

Let $\boldsymbol{\beta}_{1}=\boldsymbol{R}_{i} \boldsymbol{\theta}-\boldsymbol{r}_{i}$ and $\boldsymbol{\beta}_{*}=\boldsymbol{R}_{i} \boldsymbol{L}^{-1} \boldsymbol{\gamma}-\boldsymbol{r}_{i}$ with

$$
\pi_{u}^{*}\left(\boldsymbol{\beta}_{1}\right)=N\left(0, \frac{\omega}{n} \boldsymbol{R}_{i} \boldsymbol{S}_{Y} \boldsymbol{R}_{i}^{T}\right),
$$


and

$$
\pi_{u}^{*}\left(\boldsymbol{\beta}_{*}\right)=N\left(0, \frac{\omega}{n} \boldsymbol{R}_{i} \boldsymbol{L}^{-1} \boldsymbol{S}_{Z}\left(\boldsymbol{R}_{i} \boldsymbol{L}^{-1}\right)^{T}\right)=N\left(0, \frac{\omega}{n} \boldsymbol{R}_{i} \boldsymbol{S}_{Y} \boldsymbol{R}_{i}^{T}\right)
$$

then we have

$$
\begin{aligned}
P\left(\boldsymbol{R}_{i} \boldsymbol{\theta}>\boldsymbol{r}_{i} \mid \pi_{u}^{*}(\boldsymbol{\theta})\right) & =P\left(\boldsymbol{\beta}_{1}>0 \mid \pi_{u}^{*}\left(\boldsymbol{\beta}_{1}\right)\right)=P\left(\boldsymbol{\beta}_{*}>0 \mid \pi_{u}^{*}\left(\boldsymbol{\beta}_{*}\right)\right) \\
& =P\left(\boldsymbol{R}_{i} \boldsymbol{L}^{-1} \boldsymbol{\gamma}>\boldsymbol{r}_{i} \mid \pi_{u}^{*}(\boldsymbol{\gamma})\right)
\end{aligned}
$$

which manifests that the complexity is invariant.

Therefore the prior distribution in (16) is used for Bayes factor computation between order constrained hypotheses in SEM. Next, the posterior distribution is specified to obtain the relative fit (12).

\subsection{Normal approximations to posterior distributions}

In order to compute Bayes factors for order constrained hypotheses in SEM models, the asymptotic normality of the posterior distribution is used based on Laplace's method (DiCiccio, Kass, Raftery, and Wasserman, 1997; Gelman, Carlin, Stern, and Rubin, 2004, pp.101-107). As elaborated in the beginning of this section, the posterior distribution only depends on the density of the data $f(\boldsymbol{X} \mid \boldsymbol{\theta}, \boldsymbol{\zeta})$ when using the vague prior in (16) while letting $\omega \rightarrow \infty$. Subsequently the posterior distribution can be approximated by:

$$
\pi_{u}(\boldsymbol{\theta} \mid \boldsymbol{X}) \approx N\left(\hat{\boldsymbol{\theta}}, \hat{\boldsymbol{\Sigma}}_{\theta}\right),
$$

where $\hat{\boldsymbol{\theta}}$ denotes the estimates of the target parameters, and $\hat{\boldsymbol{\Sigma}}_{\theta}$ is their covariance matrix. Both of them can be obtained in lavaan using estimation methods, such as least square estimation and maximum likelihood estimation (Rosseel, 2012). Furthermore, to obtain standardized $\hat{\boldsymbol{\theta}}$ and $\hat{\boldsymbol{\Sigma}}_{\theta}$ lavaan provides approaches to standardize the observed variables and to directly standardize the target parameters. The performance of these two approaches of standardization was discussed in Gu et al. (2014), which showed that the variances of standardized parameters obtained using two approaches are different, whereas the resulting Bayes factors are similar. Now that the prior and posterior distributions have been specified, the Bayes factor can be obtained using (10). In the following section an efficient algorithm is described for the computation of the prior and posterior probability that the order constraints hold under $H_{u}$, which are key ingredients of the computation of the Bayes factor.

The normal approximation is widely used in hypothesis testing and model selection. Examples include Akaike's information criterion (AIC; Akaike, 
1973), Bayesian information criterion (BIC; Schwarz, 1978), and Wald's test (Gourieroux, Holly, and Monfort, 1982). In a way, the Bayes factor based on the approximated normal posterior (20) is similar as the BIC. The BIC is a large sample approximation of the marginal likelihood, whereas the proposed Bayes factor is a large sample approximation of a specific expression of the Bayes factor for an order constrained hypothesis against an unconstrained hypothesis. Both methods rely on a minimally informative prior and a large sample approximation of the posterior. An important difference is however that the proposed Bayes factor is suitable for evaluating hypotheses with order constraints while the BIC is not. To achieve this the proposed Bayes factor also needs the estimated Fisher information covariance matrix to approximate the posterior probability that the order constraints hold.

\section{An efficient algorithm for Bayes factor computa- tion}

As was elaborated in Section 3, the Bayes factor is a ratio of the posterior probability that the order constraints of $H_{i}$ hold under $H_{u}$, denoted by the relative fit $f_{i}$, and the prior probability that the order constraints of $H_{i}$ hold under $H_{u}$, denoted by the relative complexity $c_{i}$. Because both the prior distribution $\pi_{u}^{*}(\boldsymbol{\theta})=N\left(0, \omega \hat{\boldsymbol{\Sigma}}_{\theta}\right)$ and the posterior distribution $\pi_{u}(\boldsymbol{\theta} \mid \boldsymbol{X}) \approx$ $N\left(\hat{\boldsymbol{\theta}}, \hat{\boldsymbol{\Sigma}}_{\theta}\right)$ are normal distributions, for notational convenience each of them can be denoted by

$$
p(\boldsymbol{\theta})=N\left(\boldsymbol{\mu}_{\theta}, \boldsymbol{\Sigma}_{\theta}\right) .
$$

Thus, the complexity and fit can be represented by the following probability

$$
P\left(H_{i}\right)=P\left(\boldsymbol{R}_{i} \boldsymbol{\theta}>\boldsymbol{r}_{i}\right)=\int_{\boldsymbol{R}_{i} \boldsymbol{\theta}>\boldsymbol{r}_{i}} p(\boldsymbol{\theta}) d \boldsymbol{\theta} .
$$

This probability can be estimated by sampling from the prior or posterior distribution using the Gibbs sampler (Gelman, et al., 2004).

Before presenting the core algorithm of the Gibbs sampler, we shall present two pre-steps of the sampling procedure which can efficiently reduce the computing time. First, the Bayes factor is decomposed in Section 5.1 such that less iterations of the Gibbs sampler are needed to accurately estimate the complexity and fit. Second, the target parameters are transformed in Section 5.2 such that in each iteration of the Gibbs sampler less time is needed. Thereafter, Section 5.3 introduces the constrained Gibbs sampling procedure based on the transformed parameters. After obtaining 
the samples of transformed parameters, decomposed complexities and fits can be estimated using two methods proposed in Section 5.4. Furthermore, the sample size of the Gibbs sampler for accurate estimation of the complexity and fit is discussed in Section 5.5. Section 5.6 summarizes the constrained Gibbs sampling procedure by which we estimate the complexity and fit, and thus the Bayes factor.

\subsection{Decomposition of the Bayes factor}

When hypothesis $H_{i}$ is formulated using a relatively large number of order constraints, accurately estimating the complexity and fit can be computationally intensive. For example, Gu et al (2014) showed that the complexity of $H_{1}: \theta_{1}>, \ldots,>\theta_{10}$ under prior

$$
\pi_{u}^{*}(\boldsymbol{\theta})=N(\mathbf{0}, \omega \mathbf{I})
$$

is $c_{1}=1 / J !=1 / 10$ !, that is, a very small value with the need of more than 20 million Gibbs sampler draws (Hoijtink, 2012, p.207) to ensure the deviation of the estimate is almost never over $10 \%$. Directly estimating this complexity may not be feasible or extremely time-consuming. This conclusion also applies to the estimation of the complexity under $\pi_{u}^{*}(\boldsymbol{\theta})$ and the fit, because the accuracy of the estimation only depends on the size of complexity or fit and the number of Gibbs sampler draws. Consequently, when computing the Bayes factor for hypotheses with relatively large $K$, a decomposition of the Bayes factor is needed (Klugkist, Laudy, and Hoijtink, 2010):

$$
B F_{i u}=B F_{i_{1}, u} \times B F_{i_{2}, i_{1}} \times \cdots \times B F_{i_{K}, i_{K-1}},
$$

where $i_{k}, k=1, \ldots, K$ denotes a hypothesis using the constraints in the first $k$ rows of $\boldsymbol{R}_{i}$. More specifically, $B F_{i_{k}, i_{k-1}}$ is defined by:

$$
B F_{i_{k}, i_{k-1}}=\frac{f_{i_{k}, i_{k-1}}}{c_{i_{k}, i_{k-1}}}
$$

Let $H_{i_{k}}$ denote the hypothesis using constraints in the first $k$ rows of $\boldsymbol{R}_{i}$, then $c_{i_{k}, i_{k-1}}$ and $f_{i_{k}, i_{k-1}}$ denote the probabilities of prior and posterior distributions in agreement with $H_{i_{k}}$ conditional on $H_{i_{k-1}}$, respectively. Then, the complexity and fit can be expressed by

$$
c_{i}=\prod_{k=1}^{K} c_{i_{k}, i_{k-1}} \text { and } f_{i}=\prod_{k=1}^{K} f_{i_{k}, i_{k-1}} .
$$


Let

$$
P\left(H_{i_{k}} \mid H_{i_{k-1}}\right)=P\left(\boldsymbol{R}_{i_{k}} \boldsymbol{\theta}>\boldsymbol{r}_{i_{k}} \mid \boldsymbol{R}_{i_{1}} \boldsymbol{\theta}>\boldsymbol{r}_{i_{1}}, \ldots, \boldsymbol{R}_{i_{k-1}} \boldsymbol{\theta}>\boldsymbol{r}_{i_{k-1}}\right)
$$

denote either $c_{i_{k}, i_{k-1}}$ or $f_{i_{k}, i_{k-1}}$, then the probability (22) for $c_{i}$ and $f_{i}$ becomes

$$
P\left(H_{i}\right)=P\left(H_{i_{1}}\right) \times P\left(H_{i_{2}} \mid H_{i_{1}}\right) \times \cdots \times P\left(H_{i_{K}} \mid H_{i_{K-1}}\right) .
$$

Because each of the probabilities in (27) is larger than $P\left(H_{i}\right)$ especially when $K$ is large, accurately estimating $c_{i_{k}, i_{k-1}}$ or $f_{i_{k}, i_{k-1}}$ requires much less draws from the Gibbs sampler compared to directly estimating $c_{i}$ or $f_{i}$. Although every probability in (27) needs to be estimated, the total sample size for decomposed $c_{i}$ or $f_{i}$ is still less than that without decomposition because the sample size for accurate estimation increases dramatically as $K$ increases. This will be illustrated in Section 5.5. Before introducing the method for the computation of the probability (26), we transform the target parameters such that the order constrained hypothesis has a simple form, which will be elaborated in the next section.

\subsection{Transformation of target parameters}

This section simplifies the form of the hypothesis $H_{i}$ using parameter transformation $\boldsymbol{\beta}=\boldsymbol{R}_{i} \boldsymbol{\theta}-\boldsymbol{r}_{i}$ such that $H_{i}: \boldsymbol{R}_{i} \boldsymbol{\theta}>\boldsymbol{r}_{i}$ becomes $H_{i}: \boldsymbol{\beta}>0$ and the decomposed complexity or fit shown in (26) becomes

$$
P\left(H_{i_{k}} \mid H_{i_{k-1}}\right)=P\left(\beta_{k} \mid \beta_{1}>0, \ldots, \beta_{k-1}>0\right) .
$$

This transformation was also used in Mulder (2016). It has three benefits in terms of the efficiency of estimating the decomposed complexity and fit. First, the subset of vector $\boldsymbol{\beta}$ that needs to be sampled has a length that is less than or equal to $J$ (the length of $\boldsymbol{\theta}$ ). Take hypothesis $H_{1}: \theta_{1}>\theta_{2}>\theta_{3}$ for example. The transformation $\left(\beta_{1}, \beta_{2}\right)^{T}=\left(\theta_{1}-\theta_{2}, \theta_{2}-\theta_{3}\right)^{T}$ leads to $H_{1}: \beta_{1}>0, \beta_{2}>0$. Therefore, we only need to sample $\boldsymbol{\beta}$ with a length of 2. Although for another example $H_{2}: \theta_{1}>0, \theta_{2}>0, \theta_{1}>\theta_{2}$ the length of $\boldsymbol{\beta}$, where $\left(\beta_{1}, \beta_{2}, \beta_{3}\right)^{T}=\left(\theta_{1}, \theta_{2}, \theta_{1}-\theta_{2}\right)^{T}$, is larger than the length of $\boldsymbol{\theta}$, only a subset $\left(\beta_{1}, \beta_{2}\right)^{T}$ needs to be sampled because $\beta_{3}=\beta_{1}-\beta_{2}$. This issue will be further explained in the following paragraph. Second, it is more straightforward to define the conditional probability in (28) than in (26), because each $\beta$ has a lower bound of 0 if it is constrained, whereas if $\theta$ is constrained, a lower and upper bound has to be determined which will take much effort especially when $K$ is relatively large. It will be shown in Section 5.3 how the constrained $\boldsymbol{\beta}$ can be sampled. Third, the conditional probability 
$P\left(\beta_{k} \mid \beta_{1}>0, \ldots, \beta_{k-1}>0\right)$ can analytically be determined, which will be further discussed in Section 5.4.

Since $\boldsymbol{\theta}$ has a multivariate normal distribution (21), after the linear transformation, $\boldsymbol{\beta}$ also has a multivariate normal distribution $p(\boldsymbol{\beta})=N\left(\boldsymbol{\mu}_{\beta}, \boldsymbol{\Sigma}_{\beta}\right)$, where $\boldsymbol{\mu}_{\beta}=\boldsymbol{R}_{i} \boldsymbol{\mu}_{\theta}-\boldsymbol{r}_{i}$ and $\boldsymbol{\Sigma}_{\beta}=\boldsymbol{R}_{i} \boldsymbol{\Sigma}_{\theta} \boldsymbol{R}_{i}^{T}$. It should be noted that if $\boldsymbol{R}_{i}$ is of full row rank, then the elements of $\boldsymbol{\beta}$ is linearly independent, otherwise the elements of $\boldsymbol{\beta}$ are not independent. Take, for example, hypothesis

$$
H_{3}: \begin{gathered}
\theta_{1}>\theta_{3} \\
\theta_{1}>\theta_{4} \\
\theta_{2}>\theta_{3} \\
\theta_{2}>\theta_{4}
\end{gathered} \text { with }\left[\boldsymbol{R}_{3} \mid \boldsymbol{r}_{3}\right]=\left(\begin{array}{cccc:c}
1 & 0 & -1 & 0 & 0 \\
1 & 0 & 0 & -1 & 0 \\
0 & 1 & -1 & 0 & 0 \\
0 & 1 & 0 & -1 & 0
\end{array}\right)
$$

The matrix $\boldsymbol{R}_{3}$ has a rank of 3 and the transformation

$$
\boldsymbol{\beta}=\left(\begin{array}{c}
\beta_{1} \\
\beta_{2} \\
\beta_{3} \\
\beta_{4}
\end{array}\right)=\boldsymbol{R}_{3} \boldsymbol{\theta}-\boldsymbol{r}_{3}=\left(\begin{array}{c}
\theta_{1}-\theta_{3} \\
\theta_{1}-\theta_{4} \\
\theta_{2}-\theta_{3} \\
\theta_{2}-\theta_{4}
\end{array}\right)
$$

implies that $\beta_{4}=-\beta_{1}+\beta_{2}+\beta_{3}$. Without loss of generality, we suppose the rank of $\boldsymbol{R}_{i}$ is $M$ and let

$$
\boldsymbol{\beta}=(\overline{\boldsymbol{\beta}}, \tilde{\boldsymbol{\beta}})=\left(\bar{\beta}_{1}, \ldots, \bar{\beta}_{M}, \tilde{\beta}_{M+1}, \ldots, \tilde{\beta}_{K}\right),
$$

where $\overline{\boldsymbol{\beta}}$ contains $M$ independent elements of $\boldsymbol{\beta}$, and $\tilde{\boldsymbol{\beta}}$ is a linear combination of the elements of $\overline{\boldsymbol{\beta}}$. This implies that we only need to sample $\overline{\boldsymbol{\beta}}$ from its distribution. The distribution of $\overline{\boldsymbol{\beta}}$ is $p(\overline{\boldsymbol{\beta}})=N\left(\boldsymbol{\mu}_{\bar{\beta}}, \boldsymbol{\Sigma}_{\bar{\beta}}\right)$ with $\boldsymbol{\mu}_{\bar{\beta}}=\overline{\boldsymbol{R}}_{i} \boldsymbol{\mu}_{\theta}-\overline{\boldsymbol{r}}_{i}$ and $\boldsymbol{\Sigma}_{\bar{\beta}}=\overline{\boldsymbol{R}}_{i} \boldsymbol{\Sigma}_{\theta} \overline{\boldsymbol{R}}_{i}^{T}$, where $\overline{\boldsymbol{R}}_{i}$ is a full row rank matrix that consists of $M$ rows of $\boldsymbol{R}_{i}$ and $\overline{\boldsymbol{r}}_{i}$ is the corresponding constant vector. Although $\overline{\boldsymbol{R}}_{i}$ may not be unique, any set of linearly independent $M$ rows of $\boldsymbol{R}_{i}$ can be chosen because the order of constraints does not affect the evaluation of the hypothesis.

The specification of $\overline{\boldsymbol{R}}_{i}, \overline{\boldsymbol{r}}_{i}$, and the linear combination of $\overline{\boldsymbol{\beta}}$ that renders $\tilde{\boldsymbol{\beta}}$ can be achieved using elementary row operations (Gaussian elimination) for the matrix $\boldsymbol{R}_{i}$. The procedure is implemented in R package Bain. Details are given as follows:

1. Set an identity matrix $\boldsymbol{C}$ with a rank of $\max \{K, J\}$. Initialize $\boldsymbol{A}=\boldsymbol{R}_{i}$, $M=K$ and $\boldsymbol{d}=(1,2, \ldots, K)$ to record the swap of constraints in $\boldsymbol{R}_{i}$.

2. Repeat step (i), (ii) and (iii) for $k=1, \cdots, K$. 
(i) If $\boldsymbol{A}_{k, k}=0$ and $\boldsymbol{A}_{k^{\prime}, k} \neq 0$ where $k^{\prime}>k$, then swap the $k$ th row with the $k^{\prime}$ th row in $\boldsymbol{A}$ and $\boldsymbol{C}$, and swap $d_{k}$ and $d_{k^{\prime}}$ in $\boldsymbol{d}$.

(ii) If $\boldsymbol{A}_{k, k} \neq 0$ after step (i), then let $\boldsymbol{A}_{k, j}=\boldsymbol{A}_{k, j} / \boldsymbol{A}_{k, k}$ and $\boldsymbol{C}_{k, j}=$ $\boldsymbol{C}_{k, j} / \boldsymbol{C}_{k, k}$ for $j=1, \cdots, J$.

(iii) Let $\boldsymbol{A}_{k^{\prime}, j}=\boldsymbol{A}_{k^{\prime}, j}-\boldsymbol{A}_{k, j} \boldsymbol{A}_{k^{\prime}, k}$ and $\boldsymbol{C}_{k^{\prime}, j}=\boldsymbol{C}_{k^{\prime}, j}-\boldsymbol{C}_{k, j} \boldsymbol{C}_{k^{\prime}, k}$ for all $k^{\prime} \neq k$ and $j=1, \cdots, J$.

3. For $k=1, \cdots, K$, if $\sum_{j=1}^{J}\left|\boldsymbol{A}_{k, j}\right|=0$ then $M=M-1$.

4. For $k=1, \cdots, K$, if $\sum_{j=1}^{J}\left|\boldsymbol{A}_{k, j}\right|=0$ and $\sum_{j=1}^{J}\left|\boldsymbol{A}_{k^{\prime}, j}\right| \neq 0$ where $k^{\prime}>k$, then swap the $k$ th row with the $k^{\prime}$ th row in $\boldsymbol{A}$ and $\boldsymbol{C}$, and swap $d_{k}$ and $d_{k^{\prime}}$ in $\boldsymbol{d}$.

5. Let $\boldsymbol{R}_{i}=\left(\boldsymbol{R}_{i, d_{1}}, \ldots, \boldsymbol{R}_{i, d_{K}}\right)^{T}$ and $\boldsymbol{r}_{i}=\left(r_{d_{1}}, \ldots, r_{d_{K}}\right)$, where $\boldsymbol{R}_{i, d_{k}}$ denotes the $d_{k}$ th row of $\boldsymbol{R}_{i}$. Then let $\boldsymbol{\beta}=\boldsymbol{R}_{i} \boldsymbol{\theta}>\boldsymbol{r}_{i}$ in which $\overline{\boldsymbol{\beta}}$ corresponds to the first $M$ elements in $\boldsymbol{\beta}$ and $\tilde{\boldsymbol{\beta}}$ corresponds to the remaining part.

After conducting this procedure, we obtain the rank of $\boldsymbol{R}_{i}$, i.e., $M$, and $\left[\overline{\boldsymbol{R}}_{i} \mid \overline{\boldsymbol{r}}_{i}\right]$ which contains the first $M$ rows of $\left[\boldsymbol{R}_{i} \mid \boldsymbol{r}_{i}\right]$. Furthermore, the dependence in $\boldsymbol{\beta}$ can be expressed by

$$
\begin{gathered}
C_{M+1, d_{1}} \cdot \beta_{1}+\cdots+C_{M+1, d_{K}} \cdot \beta_{K}=r_{d_{M+1}}, \\
\vdots \\
C_{K, d_{1}} \cdot \beta_{1}+\cdots \\
\cdots
\end{gathered}
$$

For example, for the hypothesis $H_{3}$ shown in (29), executing the procedure above renders

$$
\begin{aligned}
{[\boldsymbol{A} \mid \boldsymbol{C}] } & =\left(\begin{array}{cccc:cccc}
1 & 0 & -1 & 0 & 1 & 0 & 0 & 0 \\
1 & 0 & 0 & -1 & 0 & 1 & 0 & 0 \\
0 & 1 & -1 & 0 & 0 & 0 & 1 & 0 \\
0 & 1 & 0 & -1 & 0 & 0 & 0 & 1
\end{array}\right) \\
& \rightarrow\left(\begin{array}{cccc:cccc}
1 & 0 & 0 & -1 & 0 & 1 & 0 & 0 \\
0 & 1 & 0 & -1 & -1 & 1 & 1 & 0 \\
0 & 0 & 1 & -1 & -1 & 1 & 0 & 0 \\
0 & 0 & 0 & 0 & 1 & -1 & -1 & 1
\end{array}\right)
\end{aligned}
$$

and $\boldsymbol{d}=(1,3,2,4)$ which means the second and third rows have been swapped. Since there are three non-zero rows in $\boldsymbol{A}$ after Gaussian elimination, the rank of $\boldsymbol{R}_{i}$ is $M=3$ and the first three rows of $\boldsymbol{R}_{i}$ are independent because they correspond to the non-zero rows. Furthermore, 
according to (32) the last row of $\boldsymbol{C}$ after Gaussian elimination indicates $\beta_{1}-\beta_{2}-\beta_{3}+\beta_{4}=0$, i.e., $\beta_{4}=-\beta_{1}+\beta_{2}+\beta_{3}$.

After the transformation of target parameters, the probability $P\left(\beta_{k} \mid \beta_{1}>\right.$ $\left.0, \ldots, \beta_{k-1}>0\right)$ from equation (28) can be estimated using the constrained Gibbs sampler. This will be discussed in the next section.

\subsection{Constrained Gibbs sampler}

The constrained Gibbs sampler is applied to estimate each decomposed complexity and fit. The basic principle of the Gibbs sampler is to sequentially generate a sample for each $\beta$ conditionally on the current values of all the others. As was elaborated before, only $\overline{\boldsymbol{\beta}}$ needs to be sampled, and the sample of $\tilde{\boldsymbol{\beta}}$ can be computed using the sample of $\overline{\boldsymbol{\beta}}$. Since $\overline{\boldsymbol{\beta}}$ is normally distributed, the conditional distribution of any parameter of $\overline{\boldsymbol{\beta}}$ given the remaining parameters is also normal. In each iteration, $\bar{\beta}_{k}^{t}$, where $t$ denotes the iteration index of the Gibbs sampler and $k=1, \ldots, M$, can be sampled from the following conditional distribution

$p\left(\bar{\beta}_{k}^{t} \mid \bar{\beta}_{l \neq k}^{t}\right)=N\left(\mu_{\bar{\beta}_{k}}+\sum_{l=1}^{k-1} b_{k l}\left(\bar{\beta}_{l}^{t}-\mu_{\bar{\beta}_{l}}\right)+\sum_{l=k+1}^{M} b_{k l}\left(\bar{\beta}_{l}^{t-1}-\mu_{\bar{\beta}_{l}}\right),\left[\left(\boldsymbol{\Sigma}_{\bar{\beta}}^{-1}\right)_{k k}\right]^{-1}\right)$,

where $\mu_{\bar{\beta}_{k}}$ is the mean of $\bar{\beta}_{k}$ in this full conditional distribution, $b_{k l}$ is the element at the $k$ th row and $l$ th column in the matrix $\boldsymbol{B}_{M \times M}=\boldsymbol{I}-$ $\left[\operatorname{diag}\left(\boldsymbol{\Sigma}_{\bar{\beta}}^{-1}\right)\right]^{-1} \boldsymbol{\Sigma}_{\bar{\beta}}^{-1}$ with $\boldsymbol{\Sigma}_{\bar{\beta}}$ being the covariance matrix of $\overline{\boldsymbol{\beta}}$ and $\boldsymbol{I}$ being a $M \times M$ identity matrix, and $\left(\boldsymbol{\Sigma}_{\bar{\beta}}^{-1}\right)_{k k}$ is the element at the $k$ th row and $k$ th column in $\boldsymbol{\Sigma}_{\bar{\beta}}^{-1}$. The derivation of equation (34) can be found in Gelman, et al. (2004, pp.579).

The estimation of probability $P\left(\beta_{k} \mid \beta_{1}>0, \ldots, \beta_{k-1}>0\right)$ requires a sample of $\overline{\boldsymbol{\beta}}=\left(\bar{\beta}_{1}, \ldots, \bar{\beta}_{M}\right)$ from the prior or posterior distribution that is in agreement with the first $k-1$ constraints $\beta_{1}>0, \ldots, \beta_{k-1}>0$. Using the current value of $\boldsymbol{\beta}$ and the linear restriction if $R_{i}$ is not of full row rank, a lower bound $L$ and a upper bound $U$ of $\overline{\boldsymbol{\beta}}$ can be specified. More specifically, if $k \leq M+1$ then $\left(\bar{\beta}_{1}, \cdots, \bar{\beta}_{k}\right)$ are sampled with a lower bound of $L=0$ and no upper bound, and other $\beta$ s are not constrained. If $k>M+1$, all $\overline{\boldsymbol{\beta}}$ have a lower bound of $L=0$, and $\left(\tilde{\beta}_{M+1}>0, \ldots, \tilde{\beta}_{k-1}>0\right)$ will be used to define a further lower bound and a upper bound of $\overline{\boldsymbol{\beta}}$ based on their dependence. Using inverse probability sampling (Gelfand, Smith, and Lee, 1992), it is straightforward to obtain a sample from truncated normal distribution (34) constrained in $(L, U)$ according to the following two steps. 
(i) Randomly generate a number $\nu$ via a uniform distribution on the interval $[0,1]$.

(ii) Compute $\bar{\beta}_{k}=\Phi_{\bar{\beta}_{k}}^{-1}\left[\Phi_{\bar{\beta}_{k}}(L)+\nu\left(\Phi_{\bar{\beta}_{k}}(U)-\Phi_{\bar{\beta}_{k}}(L)\right)\right]$, where $\Phi_{\bar{\beta}_{k}}$ is the cumulative distribution function of (34) and $\Phi_{\bar{\beta}_{k}}^{-1}$ is the inverse cumulative distribution function.

Running the Gibbs sampler for $t=1, \ldots, T$ iterations renders a sample of each component of $\overline{\boldsymbol{\beta}}=\left(\bar{\beta}_{1}, \ldots, \bar{\beta}_{M}\right)$. As elaborated in Section $5.2, \tilde{\boldsymbol{\beta}}$ is linearly dependent on $\overline{\boldsymbol{\beta}}$. Thus, we can also obtain a sample of $\tilde{\boldsymbol{\beta}}$ using the sample of $\overline{\boldsymbol{\beta}}$ and equation (32).

The choice of burn-in period and the check of convergence are important steps in the Gibbs sampler. In our method, however, we specify the prior distribution and approximate the posterior distribution with a multivariate normal distribution. Therefore, convergence is not an issue because the sample from multivariate normal distribution converges very fast even if the initial value is far away from the prior or posterior mode. This is explicitly illustrated in $\mathrm{Gu}$ et al. (2014), which applies the constrained Gibbs sampler to multivariate normal distributions as well. In addition, $\mathrm{Gu}$ et al. (2014) also shows that within a burn-in period of 100 iterations the effect of the initial values is eliminated and the sample converges to the desired distribution. Thus, we discard the first 100 iterations as a burn-in phase of the Gibbs sampler. In the next section, two methods for estimating the decomposed complexity and fit are presented based on the samples of $\boldsymbol{\beta}$ obtained in this section.

\subsection{Two methods for estimating complexity and fit}

In this section, we propose two approaches to estimate the probability (28) after obtaining the samples of $\boldsymbol{\beta}$ of size $T$ from either prior or posterior distribution. A straightforward manner is counting the number of samples in agreement with $\beta_{k}>0$ :

$$
P\left(\beta_{k}>0 \mid \beta_{1}>0, \ldots, \beta_{k-1}>0\right)=T^{-1} \sum_{t=1}^{T} I\left(\beta_{k}^{t}>0 \mid \beta_{1}^{t}>0, \ldots, \beta_{k-1}^{t}>0\right),
$$

where $I(\cdot)$ denotes the indicator function which is 1 if the argument is true and 0 otherwise.

Particularly for estimating this probability with respect to the first $M$ decomposed constraints $\overline{\boldsymbol{\beta}}>0$, we adopt a more efficient approach inspired 
by Gelfand and Smith (1992) and used in Morey, Rouder, Pratte, and Speckman (2011), and Mulder (2016). The principle of this method is that the density of the univariate $\beta_{k}$ can be approximated by the average of its full conditional density constructed using the current sample of all the other $\beta$ s. This implies the probability $P\left(\beta_{k}>0\right)$ given the density of $\beta_{k}$ can be approximated by the average of $P\left(\beta_{k}>0\right)$ given the conditional density based on different samples. Consequently, using the constrained samples for $\beta_{1}, \ldots, \beta_{k-1}$ in the conditional density, we obtain

$$
\begin{aligned}
& P\left(\beta_{k}>0 \mid \beta_{1}>0, \ldots, \beta_{k-1}>0\right) \\
= & T^{-1} \sum_{t=1}^{T} P\left(\beta_{k}>0 \mid \beta_{1}^{t}>0, \ldots, \beta_{k-1}^{t}>0, \beta_{k+1}^{t}, \ldots, \beta_{K}^{t}\right) .
\end{aligned}
$$

This probability can easily be computed because the conditional distribution (34) of $\beta_{k}$ is a univariate normal distribution that is easily integrated for $\beta_{k}>0$.

It should be emphasised that this method is not applicable for estimating decomposed complexities or fits for which $k>M$, because $\tilde{\beta}_{k}$ for $k>M$ is a linear combination of $\bar{\beta}_{1}, \ldots, \bar{\beta}_{M}$, which means $\tilde{\beta}_{k}$ is a point given $\bar{\beta}_{1}, \ldots, \bar{\beta}_{M}$. Therefore in this case equation (35) will be used. Despite of this limitation, the new method (36) is still attractive because it increases the accuracy of the estimation for a give sample size of the Gibbs sampler. This will be elaborated in the next paragraph. This implies that fewer iterations of the Gibbs sampler are needed to obtain an acceptable accuracy. Consequently, for estimating the decomposed complexities and fits in our program, the new method (36) is used when $k \leq M$, whereas the approach shown in (35) is used when $k>M$.

To investigate the performance of the two methods, we shall consider a series of hypotheses $H_{1}: \theta_{1}>\ldots>\theta_{J}$ for $J=3, \ldots, 5$ and estimate the complexities under $\pi_{u}^{*}(\theta)=N\left(\mathbf{0}, \omega \hat{\boldsymbol{\Sigma}}_{\theta}\right)$, where $\mathbf{0}$ is a zero vector with a length of $J, \hat{\boldsymbol{\Sigma}}_{\theta}=\boldsymbol{I}$ is a $J \times J$ identity matrix, and $\omega \rightarrow \infty$. The true value of $c_{1}$ with respect to prior $\pi_{u}^{*}(\theta)$ in these hypotheses is known as $c_{1}^{\text {True }}=1 / J$ !. We estimate the complexities of $H_{1} 1000$ times using each method when the sample size of the Gibbs sampler is $T=50,500$, and 5000. This results in $c_{11}^{(s)}$ and $c_{12}^{(s)}$ based on methods (35) and (36), respectively, where $s=1, \ldots, 1000$. Thereafter, we compute the mean squared relative error (MSRE), $M S R E_{1}=$ $\frac{1}{1000} \sum_{s=1}^{1000}\left(\frac{c_{1}^{\text {True }}-c_{11}^{(s)}}{c_{1}^{\text {True }}}\right)^{2}$ and $M S R E_{2}=\frac{1}{1000} \sum_{s=1}^{1000}\left(\frac{c_{1}^{\text {True }}-c_{12}^{(s)}}{c_{1}^{\text {True }}}\right)^{2}$, to measure the accuracy of the estimation using methods (35) and (36), respectively.

Table 1 displays the MSREs of the estimate for $c_{1}$. As can be seen in Ta- 
Table 1: MSRE of estimate using two methods

\begin{tabular}{|c|c|c|c|c|c|c|}
\hline \multirow[t]{2}{*}{ True } & \multicolumn{2}{|c|}{$c_{i}=0.166(\mathrm{~J}=3)$} & \multicolumn{2}{|c|}{$c_{i}=4.17 \mathrm{E}-2(\mathrm{~J}=4)$} & \multicolumn{2}{|c|}{$c_{i}=8.33 \mathrm{E}-3(\mathrm{~J}=5)$} \\
\hline & $S_{1}$ & $\mathrm{MS}$ & $\mathrm{MSRE}_{1}$ & MSI & $\mathrm{MS}$ & $2 \mathrm{E}_{2}$ \\
\hline & & 3.3 & 0 & 3. & 0.2 & $\overline{E-2}$ \\
\hline$T=$ & 3 & 7. & $61 \mathrm{~T}$ & -3 & -2 & E-3 \\
\hline$T=5000$ & $5.28 \mathrm{E}-4$ & $7.78 \mathrm{E}-5$ & $1.49 \mathrm{E}-3$ & $3.38 \mathrm{E}-4$ & $2.46 \mathrm{E}-3$ & $9.15 \mathrm{E}-4$ \\
\hline
\end{tabular}

ble 1, the MSREs from method (36) $\mathrm{MSRE}_{2}$ are much smaller than that from method (35) MSRE ${ }_{1}$. This implies that method (36) needs a smaller sample size of the Gibbs sampler to attain the same accuracy. Furthermore, it can be seen that the MSREs decreases as sample size increases, and small complexity $c_{i}=8.33 \mathrm{E}-3$ needs more sample size than large complexity $c_{i}=0.166$ to obtain the same magnitude of MSREs. This implies we can determine sample size $T$ for both methods (35) and (36) based on the acceptable estimation accuracy and the size of the probability under estimation. This will be discussed in the next section.

\subsection{Sample size determination for the Gibbs sampler}

This section discusses the sample size $T$ of the Gibbs sampler needed to accurately estimate $P\left(\beta_{k}>0 \mid \beta_{1}>0, \ldots, \beta_{k-1}>0\right)$, which has a true value $P^{\text {True }}$. As stated earlier, this probability is estimated using method (35) if $k>M$, and method (36) if $k \leq M$. For method (35), Hoijtink (2012, p.154) proposes a rule to determine the sample size $T_{1}$ needed to accurately estimate the complexity or fit, which is shown in the top panel of Table 2 . The criterion is that the $95 \%$ central credibility interval for the estimate has lower and upper bounds that are less than $10 \%$ different from the true value. The first row in Table 2 displays the true probabilities $P^{\text {True }}$ that needs to be estimated. In addition, L-95\% and U-95\% demonstrate the lower and upper bounds of the $95 \%$ central credibility interval when using the corresponding $T_{1}$ above.

For method (36), we present a new rule to determine the sample size $T_{2}$ based on a more strict accuracy criterion, that is, the differences between both L-95\% and U-95\%, and $P^{\text {True }}$ are less than $5 \%$. We let $N\left(\mu_{\beta_{k}}, \sigma_{\beta_{k}}^{2}\right)$ denote the distribution of $\beta_{k}$ in $P\left(\beta_{k} \mid \beta_{1}>0, \ldots, \beta_{k-1}>0\right)$, where $\mu_{\beta_{k}}$ is the mean and $\sigma_{\beta_{k}}^{2}$ is the variance. Then equation (36) becomes

$$
\begin{aligned}
P\left(\beta_{k} \mid \beta_{1}>0, \ldots, \beta_{k-1}>0\right) & =P\left(\beta_{k}>0 \mid \beta_{k} \sim N\left(\mu_{\beta_{k}}, \sigma_{\beta_{k}}^{2}\right)\right) \\
& =P\left(\beta_{k}>0 \mid \beta_{k} \sim N\left(\hat{\lambda}_{k}, 1\right)\right),
\end{aligned}
$$


Table 2: Gibbs sample size determination

\begin{tabular}{ccccccc}
\hline$P^{\text {True }}$ & 0.166 & $4.17 \mathrm{E}-2$ & $8.33 \mathrm{E}-3$ & $1.39 \mathrm{E}-3$ & $1.98 \mathrm{E}-4$ & $2.48 \mathrm{E}-5$ \\
\hline$T_{1}$ & 3,000 & 9,600 & 120,000 & 360,000 & $2,520,000$ & $20,160,000$ \\
$\mathrm{~L}-95 \%$ & 0.154 & $3.8 \mathrm{E}-2$ & $7.8 \mathrm{E}-3$ & $1.27 \mathrm{E}-3$ & $1.82 \mathrm{E}-4$ & $2.3 \mathrm{E}-5$ \\
$\mathrm{U}-95 \%$ & 0.180 & $4.6 \mathrm{E}-2$ & $8.9 \mathrm{E}-3$ & $1.52 \mathrm{E}-3$ & $2.17 \mathrm{E}-4$ & $2.7 \mathrm{E}-5$ \\
\hline$T_{2}$ & 4,000 & 8,000 & 12,000 & 18,000 & 25,000 & 32,000 \\
$\mathrm{~L}-95 \%$ & 0.159 & $3.97 \mathrm{E}-2$ & $7.93 \mathrm{E}-3$ & $1.32 \mathrm{E}-3$ & $1.89 \mathrm{E}-4$ & $2.37 \mathrm{E}-5$ \\
$\mathrm{U}-95 \%$ & 0.175 & $4.36 \mathrm{E}-2$ & $8.74 \mathrm{E}-3$ & $1.46 \mathrm{E}-3$ & $2.08 \mathrm{E}-4$ & $2.60 \mathrm{E}-5$ \\
\hline
\end{tabular}

where $\hat{\lambda}_{k}=\mu_{\beta_{k}} / \sigma_{\beta_{k}}$ is the standardized population mean of $\beta_{k}$. The principle of the sample size determination for method (36) is based on two facts. First, in the Gibbs sampler, we obtain $T_{2}$ samples of $\beta_{k}$ from $N\left(\mu_{\beta_{k}}, \sigma_{\beta_{k}}^{2}\right)$ or standardized $\beta_{k}$ from $N\left(\hat{\lambda}_{k}, 1\right)$. This implies that the distribution of the standardized sample mean of $\beta_{k}$, denoted by $\lambda_{k}$, is $N\left(\hat{\lambda}_{k}, \frac{1}{T_{2}}\right)$. Second, the probability $P\left(\beta_{k} \mid \beta_{1}>0, \ldots, \beta_{k-1}>0\right)$ is a one-to-one correspondence function of $\hat{\lambda}_{k}$. For example, if $\hat{\lambda}_{k}=0$, we obtain a probability of $1 / 2$, and conversely if the true value of the probability is $1 / 6$, we would expect a $\hat{\lambda}_{k}$ of -0.97 . These enable us to determine the sample size $T_{2}$ needed to accurately estimate $P\left(\beta_{k}>0 \mid \beta_{1}>0, \ldots, \beta_{k-1}>0\right)$ given a true value $P^{\text {True }}$ using the following steps.

1. Compute $\hat{\lambda}_{k}$ such that $P\left(\beta_{k}>0 \mid \beta_{k} \sim N\left(\hat{\lambda}_{k}, 1\right)\right)=P^{\text {True }}$, and initialize $T_{2}=1000$.

2. Sample $\lambda_{k} 10000$ times from $N\left(\hat{\lambda}_{k}, \frac{1}{T_{2}}\right)$, and then obtain 10000 estimates of $P\left(\beta_{k}>0 \mid \beta_{k} \sim N\left(\hat{\lambda}_{k}, 1\right)\right)$.

3. Using 10000 estimates of $P\left(\beta_{k}>0 \mid \beta_{k} \sim N\left(\hat{\lambda}_{k}, 1\right)\right)$, compute their $95 \%$ central credibility interval $(L, U)$.

4. If either $\frac{\left|L-P^{\text {True }}\right|}{P^{\text {True }}}>5 \%$ or $\frac{\left|U-P^{\text {True }}\right|}{P^{\text {True }}}>5 \%$, then $T_{2}=T_{2}+1000$ and go to Step 2.

The bottom panel of Table 2 displays the sample size $T_{2}$ and the resulting L-95\% and U-95\% from the procedure above given corresponding $P^{\text {True }}$.

In Bain, Table 2 is adopted to determine the sample size $T_{1}$ and $T_{2}$ of the Gibbs sampler for estimating each decomposed complexity and fit based on methods (35) and (36). Because $T_{1}$ or $T_{2}$ is large enough to accurately estimate the corresponding $P^{\text {True }}$ in the first row of Table 2, it will also 
be sufficient to estimate a probability that is larger than this $P^{\text {True }}$. We estimate $P\left(\beta_{k} \mid \beta_{1}>0, \ldots, \beta_{k-1}>0\right)$ with a starting sample size $T_{1}=3000$ if $k>M$ or $T_{2}=4000$ if $k \leq M$, and gradually reset $T_{1}$ or $T_{2}$ based on Table 2 until the estimate of the complexity or fit is larger than the corresponding $P^{\text {True }}$. Note that if the estimate is still less than $2.48 \mathrm{E}-5$ when using the corresponding $T_{1}$ or $T_{2}$, we specify $T_{1}=100,000,000$ or $T_{2}=100,000$.

\subsection{Summary of the computation of the Bayes factor}

This section summarizes the computation of the Bayes factor for $H_{i}$ against $H_{u}$, which is a ratio of the fit and complexity. The following steps describe how our program computes the complexity and fit, and therefore the Bayes factor.

1. Transform $\boldsymbol{\theta}$ into $\boldsymbol{\beta}$ using the procedure shown in Section 5.2. Then, we obtain $(\overline{\boldsymbol{\beta}}, \tilde{\boldsymbol{\beta}})$ and $M$ the rank of $\boldsymbol{R}_{i}$.

2. Repeat Step $(1), \ldots,(6)$ for $k=1, \ldots, K$ to estimate each $P\left(\beta_{k}>\right.$ $\left.0 \mid \beta_{1}>0, \ldots, \beta_{k-1}>0\right)$ for the decomposed complexity $c_{i_{k}, i_{k-1}}$ and fit $f_{i_{k}, i_{k-1}}$.

(1) Initialize the sample size of the Gibbs sampler as $T_{2}=4000$ if $k \leq M$ and $T_{1}=3000$ if $k>M$, and initialize $\boldsymbol{\beta}=0$.

(2) Repeat Step (a) or (b) for $t=1, \ldots, T_{2}+100$ iterations if $k \leq M$ or for $t=1, \ldots, T_{1}+100$ iterations if $k>M$, where 100 denotes the first 100 iterations, that is, a burn-in phase of the Gibbs sampler.

(a) If $k \leq M+1$, then define a boundary $(L, U)=(0, \infty)$ for $\bar{\beta}_{1}, \ldots, \bar{\beta}_{k-1}$ and no boundary for $\bar{\beta}_{k}, \ldots, \bar{\beta}_{K}$. Thereafter, sequentially generate a sample of $\overline{\boldsymbol{\beta}}^{t}$ from the truncated distribution of (34) as previously described in Step (i) and (ii) in Section 5.3.

(b) If $k>M+1$, then define a boundary $(L, U)$ for $\bar{\beta}_{1}, \ldots, \bar{\beta}_{M}$ using the linear relation between the $\overline{\boldsymbol{\beta}}>0$ and $\tilde{\boldsymbol{\beta}}>0$. Thereafter, sequentially generate a sample of $\overline{\boldsymbol{\beta}}^{t}$ from the truncated distribution of (34) as previously described in Step (i) and (ii) in Section 5.3. Then a sample of $\tilde{\boldsymbol{\beta}}^{t}$ is obtained by means of its linear dependence on $\overline{\boldsymbol{\beta}}^{t}$.

(3) Discard all the iterations for which $t \leq 100$ to account the burn-in period as discussed in Section 5.3. 
(4) If $k \leq M$, compute the probability $P\left(\beta_{k}>0 \mid \beta_{1}>0, \ldots, \beta_{k-1}>0\right)$ $=T_{2}^{-1} \sum_{t=101}^{T_{2}+100} P\left(\beta_{k}>0 \mid \beta_{k} \sim N\left(\mu_{\beta_{k}}^{t},\left(\sigma_{\beta_{k}}^{2}\right)^{t}\right)\right)$ using method (36) in Section 5.4.

(5) If $k>M$, compute the probability $P\left(\beta_{k}>0 \mid \beta_{1}>0, \ldots, \beta_{k-1}>0\right)$ $=T_{1}^{-1} \sum_{t=101}^{T_{1}+100} I\left(\beta_{k}^{t}>0 \mid \beta_{1}^{t}>0, \ldots, \beta_{k-1}^{t}>0\right)$ using method (35) in Section 5.4.

(6) If $P\left(\beta_{k} \mid \beta_{1}>0, \ldots, \beta_{k-1}>0\right)$ obtained in Step (4) or (5) is less than the reference value that corresponds to the current $T_{2}$ or $T_{1}$ in Table 2 , respectively, then reset $T_{2}$ or $T_{1}$ using the value of the next column in the table and restart the procedure from Step (2). If not, the estimation of $P\left(\beta_{k} \mid \beta_{1}>0, \ldots, \beta_{k-1}>0\right)$ is completed, which renders the decomposed complexity $c_{i_{k}, i_{k-1}}$ or fit $f_{i_{k}, i_{k-1}}$. This was elaborated in Section 5.5.

3. The complexity and fit can be computed by $c_{i}=\prod_{k=1}^{K} c_{i_{k}, i_{k-1}}$ and $f_{i}=\prod_{k=1}^{K} f_{i_{k}, i_{k-1}}$ shown in Section 5.1. Then, the Bayes factor for $H_{i}$ against $H_{u}$ is $B F_{i u}=f_{i} / c_{i}$.

\section{Empirical applications in SEM}

In this section, our procedure of evaluating order constrained hypotheses will be illustrated using two classic SEM applications. One example concerns confirmatory factor analysis (CFA), and the other example concerns multiple regression model.

\subsection{Confirmatory factor analysis}

In the first example, we reanalyze a dataset built into lavaan called HolzingerSwineford1939 (Rosseel, 2012). This dataset is taken from the Holzinger and Swineford 1939 (H\&S) study, which is a commonly used example in factor analysis. The raw dataset consists of scores of 301 seventh and eighth grade students from the Pasteur School $(\mathrm{n}=145)$ and Grant-White School $(\mathrm{n}=156)$ who participated in 26 psychological aptitude tests. In our example, only a subset with 9 variables of the complete data is extracted to measure 3 correlated latent variables, each with three indicators, i.e.,

- a visual factor $\left(\xi_{1}\right)$ is measured by visual perception $\left(x_{1}\right)$, cubes $\left(x_{2}\right)$ and lozenges $\left(x_{3}\right)$.

- a textual factor $\left(\xi_{2}\right)$ is measured by paragraph comprehension $\left(x_{4}\right)$, sentence completion $\left(x_{5}\right)$ and word meaning $\left(x_{6}\right)$ 
Table 3: Descriptives for the variables in the confirmatory factor analysis

\begin{tabular}{cccc}
\hline Variable & & Mean & S.D. \\
\hline visual perception & $x_{1}$ & 4.94 & 1.17 \\
cubes & $x_{2}$ & 6.09 & 1.18 \\
lozenges & $x_{3}$ & 2.25 & 1.13 \\
paragraph & $x_{4}$ & 3.06 & 1.16 \\
sentence & $x_{5}$ & 4.34 & 1.29 \\
word mean & $x_{6}$ & 2.19 & 1.10 \\
addition & $x_{7}$ & 4.19 & 1.09 \\
dots & $x_{8}$ & 5.53 & 1.01 \\
straight curved & $x_{9}$ & 5.37 & 1.01 \\
\hline
\end{tabular}

- a speed factor $\left(\xi_{3}\right)$ is measured by addition $\left(x_{7}\right)$, counting of dots $\left(x_{8}\right)$ and discrimination of straight and curved capitals $\left(x_{9}\right)$.

The descriptives for the observed variables are given in Table 3, whereas the relations between latent variables and their indicators are formulated in the next paragraph and expressed using path notation (without showing measurement errors) in Figure 1.

The confirmatory factor analysis model for the H\&S data can be represented as:

$$
\boldsymbol{x}=\boldsymbol{\Lambda}_{x} \boldsymbol{\xi}+\boldsymbol{\epsilon}_{x},
$$

where $\boldsymbol{x}=\left(x_{1}, \ldots, x_{9}\right)^{T}$ denotes observed variables, $\boldsymbol{\xi}=\left(\xi_{1}, \xi_{2}, \xi_{3}\right)^{T}$ denotes latent variables,

$$
\boldsymbol{\Lambda}_{x}^{T}=\left(\begin{array}{ccccccccc}
\theta_{1} & \theta_{2} & \theta_{3} & 0 & 0 & 0 & 0 & 0 & 0 \\
0 & 0 & 0 & \theta_{4} & \theta_{5} & \theta_{6} & 0 & 0 & 0 \\
0 & 0 & 0 & 0 & 0 & 0 & \theta_{7} & \theta_{8} & \theta_{9}
\end{array}\right)
$$

is a matrix of factor loadings, and $\boldsymbol{\epsilon}_{x}$ is a $3 \times 1$ vector of measurement errors with $\boldsymbol{\epsilon}_{x} \sim N\left(0, \boldsymbol{\Psi}_{\epsilon_{x}}\right)$ and $\boldsymbol{\Psi}_{\epsilon_{x}}$ being its covariance matrix. The covariance matrix of observed variables is given by:

$$
\boldsymbol{\Sigma}_{x}=\boldsymbol{\Lambda}_{x} \boldsymbol{\Phi}_{\xi} \boldsymbol{\Lambda}_{x}^{T}+\boldsymbol{\Psi}_{\epsilon_{x}}
$$

where the factor covariance matrix $\boldsymbol{\Phi}_{\xi}$ is a symmetric matrix:

$$
\boldsymbol{\Phi}_{\xi}=\left(\begin{array}{lll}
\phi_{11} & \phi_{12} & \phi_{13} \\
\phi_{12} & \phi_{22} & \phi_{23} \\
\phi_{13} & \phi_{23} & \phi_{33}
\end{array}\right)
$$




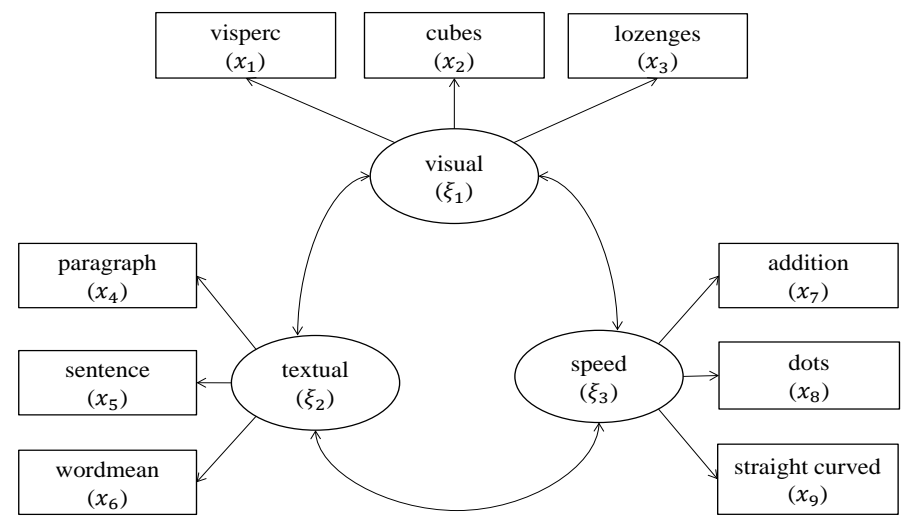

Figure 1: Confirmatory factor analysis

Because the confirmatory factor analysis model is a measurement model without a structural model, we can simply specify this model using lavaan syntax in R (see Appendix A). To ensure that the target parameters are comparable, we standardize them all. As is elaborated in Appendix A, lavaan provides both the standardized estimates and covariance matrix of target parameters. Recall that this is all the information that Bain needs to compute Bayes factors. Furthermore, in factor analysis models, indicators are required to both identify the model and set a metric for latent variables. This can be typically achieved either by standardizing the variances of latent variables or by constraining one factor loading per latent variable to 1 . In this example, the former way is chose.

Factor loadings indicate the degree of correspondence between the factor and the indicator, with higher loadings making the indicator more representative of the factor. Researchers might be interested in the issue which indicator plays the most important role in defining a factor. For instance, the first indicator of every factor may be expected to be strongest, which can be represented by the following hypothesis

$$
\begin{aligned}
\theta_{1} & >\left\{\theta_{2}, \theta_{3}\right\} \\
H_{1}: & \theta_{4}>\left\{\theta_{5}, \theta_{6}\right\} . \\
& \theta_{7}>\left\{\theta_{8}, \theta_{9}\right\}
\end{aligned}
$$


We can also test a hypothesis with respect to the structure of the correlations between the latent variables. For example, we can evaluate whether the correlation between visual and textual is larger than the correlation either between visual and speed or between textual and speed:

$$
H_{2}: \phi_{12}>\left\{\phi_{13}, \phi_{23}\right\}
$$

Using R package Bain (the user manual of Bain can be found in Appendix B) to compute Bayes factors for $H_{1}$ against $H_{u}$ or $H_{1_{c}}$ renders $B F_{1 u}=0.076$ or $B F_{11_{c}}=0.073$. For $H_{2}$ against $H_{u}$ or $H_{2}$, Bain renders $B F_{2 u}=1.33$ or $B F_{22_{c}}=1.59$. These results imply that hypothesis $H_{1}$ is not supported by the data, and the evidence from the data for $\mathrm{H}_{2}$ is not convincing because $B F_{2 u}$ or $B F_{22_{c}}$ is quite close to 1 .

\subsection{Multiple regression with latent variables}

In a study reported by Warren, White, and Fuller (1974) (data available at https://informative-hypotheses.sites .uu.nl/software/bain), a sample of 98 managers of farmer cooperatives was selected with the objective of studying managerial behavior. They postulated that a latent variable manager performance $(\eta)$ was predicted by three correlated latent variables, i.e., knowledge $\left(\xi_{1}\right)$, orientation $\left(\xi_{2}\right)$ and satisfaction $\left(\xi_{3}\right)$, and an observed variable training $\left(x_{4}\right)$. The latent variables $\eta, \xi_{1}, \xi_{2}$, and $\xi_{3}$ were measured based on qualitative and quantitative answers to identical questionnaires collected from a random sample of managers in farmer cooperatives. These variables are assumed to be measured with error, and the errors of measurement were computed using the split halves procedure (Warren, et al., 1974) for all variables subject to measurement error:

- $\eta$ is measured by $y_{1}$ and $y_{2}$,

- $\xi_{1}$ is measured by $x_{11}$ and $x_{12}$,

- $\xi_{2}$ is measured by $x_{21}$ and $x_{22}$,

- $\xi_{3}$ is measured by $x_{31}$ and $x_{32}$.

The observed variables are described in Table 4 and the graphical specification of this structural equation model is found in Figure 2.

As can be seen from Figure 2, the relations of the variables can be represented by a multiple regression model with $\eta, \xi_{1}, \xi_{2}$, and $\xi_{3}$ that are latent. 
Table 4: Descriptives for the variables in the multiple regression model

\begin{tabular}{ccc}
\hline Variable & Mean & S.D. \\
\hline$y_{1}$ & 1.06 & 0.16 \\
$y_{2}$ & 1.05 & 0.15 \\
$x_{11}$ & 1.43 & 0.30 \\
$x_{12}$ & 1.33 & 0.24 \\
$x_{21}$ & 2.84 & 0.43 \\
$x_{22}$ & 2.91 & 0.38 \\
$x_{31}$ & 2.54 & 0.34 \\
$x_{32}$ & 2.47 & 0.32 \\
$x_{4}$ & 2.12 & 0.31 \\
\hline
\end{tabular}

The measurement model is given by

$$
\begin{aligned}
& \boldsymbol{y}=\Lambda_{y} \eta+\boldsymbol{\epsilon}_{y} \\
& \boldsymbol{x}=\Lambda_{x} \boldsymbol{\xi}+\boldsymbol{\epsilon}_{x},
\end{aligned}
$$

where $\boldsymbol{x}=\left(x_{11}, x_{12}, x_{21}, x_{22}, x_{31}, x_{32}\right)^{T}$ denotes observed variables, and $\eta$ and $\boldsymbol{\xi}=\left(\xi_{1}, \xi_{2}, \xi_{3}\right)^{T}$ are latent variables. For the structural model, we have

$$
\eta=\theta_{0}+\theta_{1} \xi_{1}+\theta_{2} \xi_{2}+\theta_{3} \xi_{3}+\theta_{4} x_{4}+\delta
$$

where $\theta_{0}$ is the intercept, $\theta_{1}, \theta_{2}, \theta_{3}$, and $\theta_{4}$ are regression coefficients, and $\delta \sim N\left(0, \sigma^{2}\right)$ is the residual. This regression model is analyzed in lavaan (see Appendix A). We standardize the coefficients to make them comparable. Using the standardized estimates and covariance matrix of these coefficients from lavaan, Bain can compute Bayes factors.

The hypothesis we evaluated is based on the results obtained by Warren et al. (1974) It states that knowledge is the strongest predictor followed by orientation, training and satisfaction. The resulting hypothesis is

$$
H_{3}: \theta_{1}>\theta_{2}>\theta_{4}>\theta_{3} \text {. }
$$

This hypothesis can be compared to, for example, knowledge is stronger than orientation followed by satisfaction and training:

$$
H_{4}: \theta_{1}>\theta_{2}>\theta_{3}>\theta_{4}
$$

and training is stronger than satisfaction followed by orientation and knowledge:

$$
H_{5}: \theta_{4}>\theta_{3}>\theta_{2}>\theta_{1}
$$




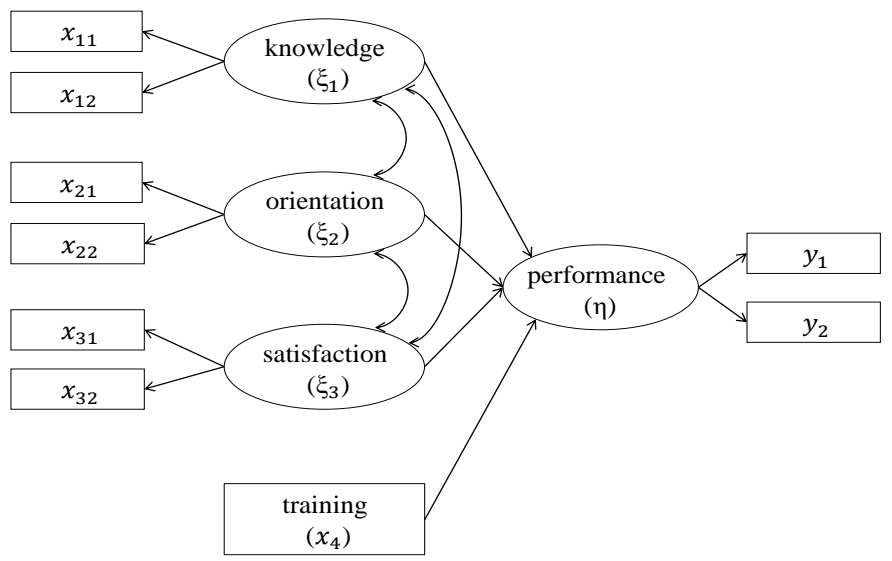

Figure 2: Multiple regression with latent variables

Table 5: Bayes factors and PMPs of $H_{3}, H_{4}$ and $H_{5}$

\begin{tabular}{ccc}
\hline & $B F_{i c}$ & PMPs \\
\hline$H_{3}$ & 11.90 & 0.785 \\
$H_{4}$ & 2.676 & 0.214 \\
$H_{5}$ & 0.010 & 0.001 \\
\hline
\end{tabular}

The results of the evaluation of these three hypotheses using Bain are displayed in Table 5 (the user manual of Bain can be found in Appendix B). As can be seen, there is evidence in favor of $H_{3}$, no convincing evidence for $H_{4}$, and evidence against $H_{5}$. Furthermore, it can be seen from the PMPs introduced in (15) that $H_{3}$ receives the largest support from the data.

\section{Discussion}

Order constrained hypotheses provide a representation of a researcher's theory with respect to the relations between the parameters of interest in SEM models. We developed a Bayes factor that can evaluate these hypotheses in a direct manner. A very vague prior was proposed that incorporates the covariance structure of the target parameters in the data. A proof was given that the prior probability that the order constraints hold, a key ingredient 
of the Bayes factor when testing order constrained hypotheses, was invariant for linear transformations of the data.

The multivariate normal prior that is used to compute the prior probability can be applied to order constrained testing problems where parameters have symmetric prior distributions such as regression coefficients, group means, and factor loadings. Even in the case of non-symmetric prior distributions, the procedure will be accurate in most cases. For example, when testing a specific ordering of $J$ variances using identical inverse gamma priors, the prior probability of this specific ordering will be equal to $1 / J$ ! which is identical to when computing the probability using independent normal priors. The method could break down in asymmetric cases where the boundary value does not lie in the middle of a parameter space. For instance when testing $0 \leq \theta<.2$ versus $.2 \leq \theta \leq 1$, where $\theta$ is the probability of a success in a binomial experiment, and a uniform prior is specified on $\theta$, the prior probabilities of $\theta$ falling in these two intervals could be different than when computing the probability using a normal distribution on $\theta$. Extending the methodology for such asymmetric testing problems would be an interesting topic to explore for future research.

Furthermore, a new algorithm was developed to ensure fast computation to ensure general utilization of the methodology by applied researchers. The methodology was implemented in the $\mathrm{R}$ package Bain which only needs the estimates and covariance matrix of target parameters (which can be obtained from the free R-package lavaan), and one or more restriction matrices representing a researcher's expectations. The output from Bain consists of Bayes factors and posterior probabilities for the hypotheses. These can be used which provide a direct answer about the relative evidence in the data between the hypotheses under investigation.

\section{Appendix}

\section{A Estimates and covariance matrix obtained using lavaan}

Bain uses the estimates and covariance matrix of target parameters to compute Bayes factors. These can be obtained from the $\mathrm{R}$ package lavaan (Rosseel, 2012). This appendix illustrates how to obtain the estimates and covariance matrix of target parameters using the two examples discussed in 
Section 6.

First of all, researchers need to install the version 0.5 -18 or higher version of lavaan by starting $R$ and typing install.packages("lavaan"). Note that $R$ should be upgraded to R.3.5.0 or a higher version. The user manual of the latest version of lavaan can be found at https: //CRAN.R-project.org/package=lavaan.

The following $R$ syntax renders the estimates and covariance matrix for the CFA model presented in Section 6.1.

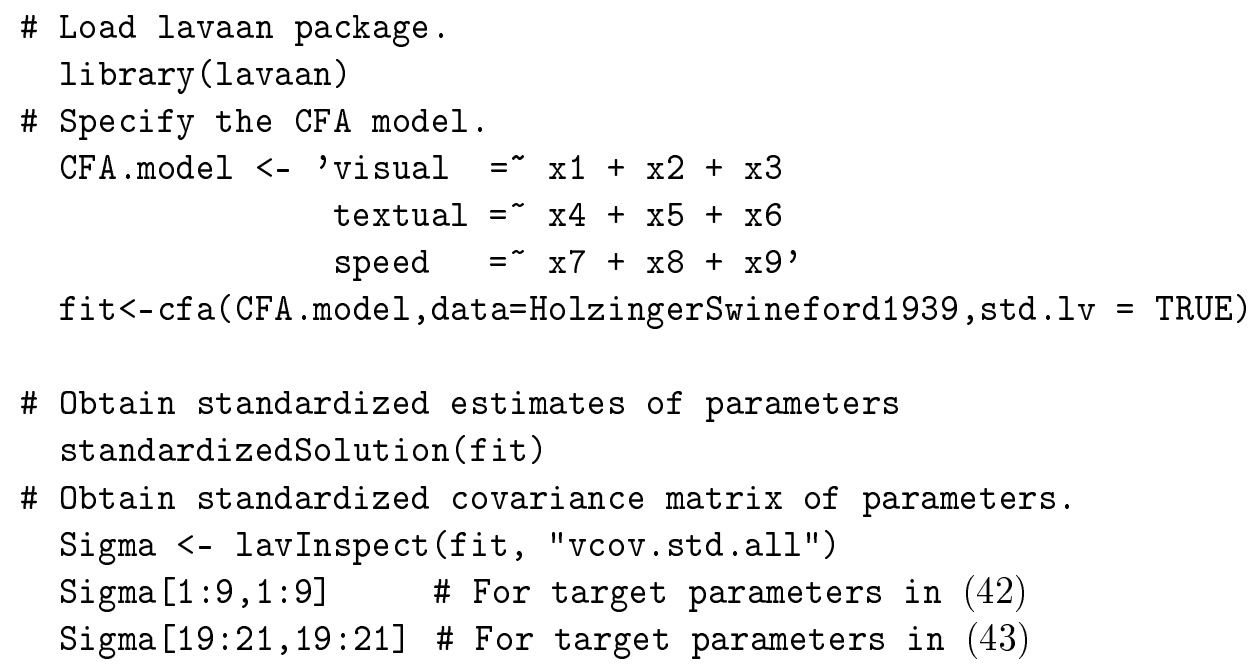

The output of standardizedSolution (fit, ci = FALSE) for the CFA model is

\begin{tabular}{|c|c|c|c|c|c|c|c|}
\hline & Ihs & $o p$ & rhs & est.std & se & $z$ & pvalue \\
\hline 1 & visual & $=\sim$ & $\mathrm{x} 1$ & 0.772 & 0.055 & 14.041 & 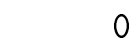 \\
\hline 2 & visual & $=^{\sim}$ & $\mathrm{x} 2$ & 0.424 & 0.060 & 7.105 & \\
\hline 3 & visual & $=^{\sim}$ & x3 & 0.581 & 0.055 & 10.539 & \\
\hline 4 & textual & $=^{\sim}$ & $\mathrm{x} 4$ & 0.852 & 0.023 & 37.776 & \\
\hline 5 & textual & $=\sim$ & $x 5$ & 0.855 & 0.022 & 38.273 & \\
\hline 6 & textual & $=^{\sim}$ & $x 6$ & 0.838 & 0.023 & 35.881 & \\
\hline 7 & speed & $=^{\sim}$ & $\mathrm{x} 7$ & 0.570 & 0.053 & 10.714 & \\
\hline$\varepsilon$ & speed & $=^{\sim}$ & $x 8$ & 0.723 & 0.051 & 14.309 & \\
\hline 9 & speed & $=\sim$ & $x 9$ & 0.665 & 0.051 & 13.015 & \\
\hline & & & & & & & \\
\hline 2 & visual & $\sim \sim$ & textual & 0.459 & 0.064 & 7.189 & \\
\hline 23 & visual & $\sim \sim$ & speed & 0.471 & 0.073 & 6.461 & \\
\hline 4 & textual & $\sim \sim$ & speed & 0.283 & 0.069 & 4.117 & \\
\hline
\end{tabular}


Note that the label visual $=^{\sim} \mathrm{x} 1$ denotes the factor loading $\theta_{1}$ relating $x_{1}$ to $\xi_{1}$ and the label visual $\sim$ textual denotes the covariance $\phi_{12}$ between $\xi_{1}$ and $\xi_{2}$. We only show the results for nine factor loadings used in (42) and three covariances used in (43). The standardized estimates of the target parameters are given in the column under est.std. For example, the estimate of $\theta_{4}$ is 0.852 in the row of textual $=^{\sim} \mathrm{x} 4$, and the estimate of $\phi_{23}$ is 0.283 in the row of textual $\sim$ speed.

The output of Sigma contains the standardized covariance matrix of the target parameters. We only show the covariance matrix Sigma $[19: 21,19: 21]$ of $\phi_{12}, \phi_{13}$, and $\phi_{23}$ :

$$
\begin{aligned}
& \text { visual textual visual } \text { speed textual } \text { speed }
\end{aligned}
$$

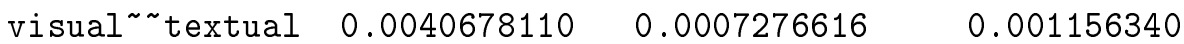

$$
\begin{aligned}
& \begin{array}{llll}
\text { textual } \sim \text { speed } & 0.0011563398 & 0.0014800678 & 0.004723718
\end{array}
\end{aligned}
$$$$
\begin{array}{llll}
\text { visual } \sim \text { speed } & 0.0007276616 & 0.0053037342 & 0.001480068
\end{array}
$$

The following $R$ syntax renders the estimates and covariance matrix for the regression model in Section 6.2.

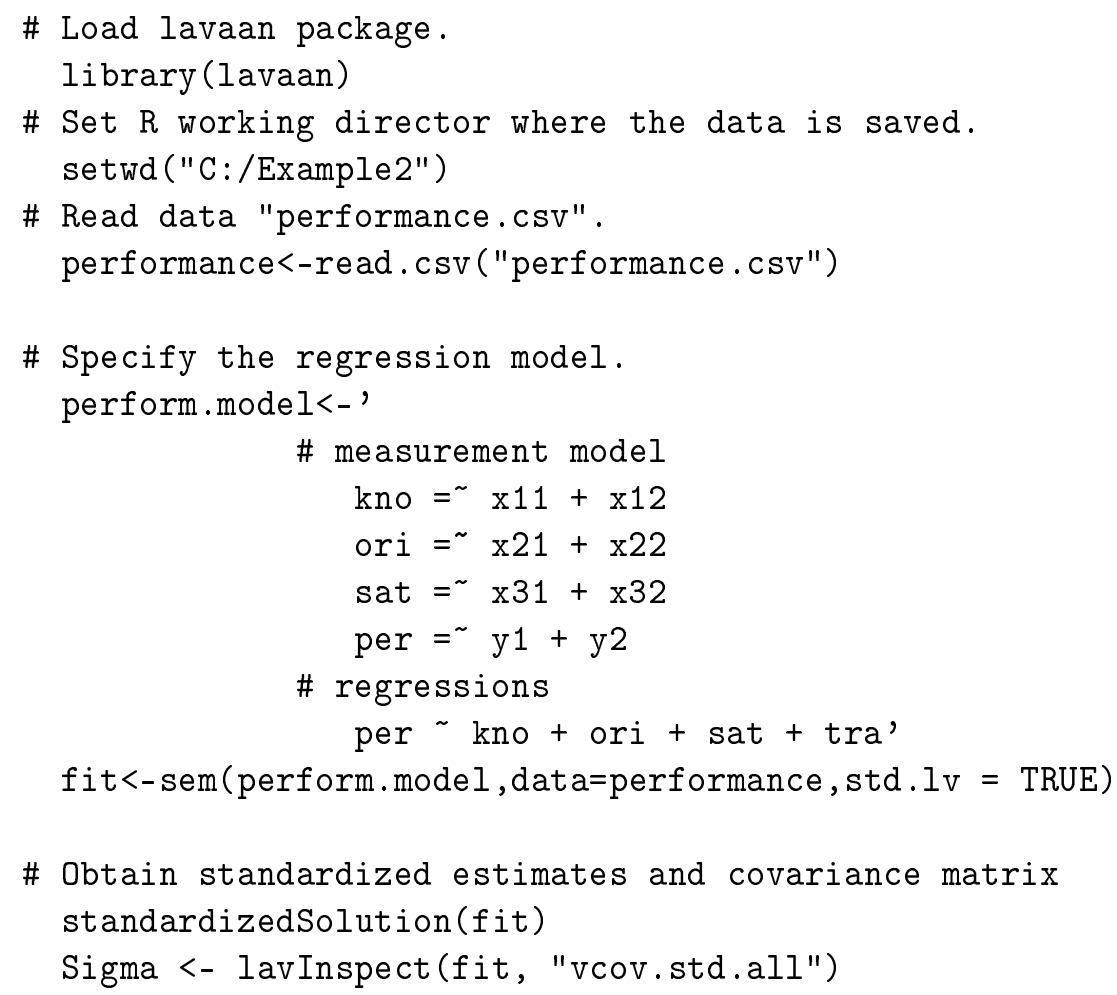


Sigma [9:12,9:12] \# For target parameters in (46), (47), (48)

The output of standardizedSolution( $f i t, c i=$ FALSE) for the regression model is

\begin{tabular}{|c|c|c|c|c|c|}
\hline lhs & op rhs & st.std & se & $z$ & pvalue \\
\hline per & $\sim \mathrm{kno}$ & 0.478 & 0.161 & 2.960 & 0.003 \\
\hline 0 per & $\sim$ ori & 0.336 & 0.165 & 2.030 & 0.042 \\
\hline per & $\sim$ sat & 0.151 & 0.105 & 1.440 & 0.150 \\
\hline 2 per & $\sim \operatorname{tra}$ & 0.286 & 0.084 & 3.403 & 0.001 \\
\hline
\end{tabular}

Note that the label per $\sim$ kno denotes the coefficient $\theta_{1}$ which relates $\eta$ to $\xi_{1}$ in the regression model (45). We only show the results for the four regression coefficients used in (46), (47), and (48). The standardized estimates of the target parameters are given in the column under est.std. For example, the estimate of $\theta_{1}$ is 0.478 in the row of per $\sim$ kno, and the estimate of $\theta_{4}$ is 0.286 in the row of per tra.

The output of Sigma $[9: 12,9: 12]$ renders the standardized covariance matrix of $\theta_{1}, \ldots, \theta_{4}$ :

$$
\text { per }{ }^{\sim} \text { pno per }{ }^{\sim} \text { ori per sat per }{ }^{\sim} \text { tra }
$$

per ${ }^{\sim}$ kno $\quad 0.026034895-0.0223249106-0.0050273595-0.0011610045$

$\begin{array}{llllll}\text { per ori }-0.022324911 & 0.0273346337 & 0.0043904540 & -0.0007619234\end{array}$

per sat $-0.005027359 \quad 0.0043904540 \quad 0.0110250662-0.0002713825$

per ${ }^{\sim}$ tra $-0.001161004-0.0007619234-0.0002713825 \quad 0.0070519650$

The standardized estimates and covariance matrix of target parameters obtained in lavaan can be used as input for Bain. This will be shown in the user manual in Appendix B.

\section{B User Manual of Bain}

Bain is an $R$ package developed for the evaluation of order constrained hypotheses using the algorithm presented in the paper. It can be downloaded at https://informative-hypotheses.sites.uu.nl/software/bain/. Windows, Mac, and Linux versions are offered, respectively, by downloaded files Bain_xxx.zip, Bain_xxx.tgz, and Bain_xxx.tar.gz, where xxx denotes package version. After downloading the package, for example, windows users can install Bain in $\mathrm{R}$ by 
install.packages("../Bain_xxx.zip", repos = NULL)

This appendix provides a brief user manual of Bain. The detailed manual can be found on the website. The core function of Bain package is

Bain (estimate, Sigma, grouppara $=0$, jointpara $=0, n$, $\mathrm{ERr}=\mathrm{NULL}, \mathrm{IRr}=\mathrm{NULL}, \ldots$, seed $=100$, print $=\mathrm{TRUE})$.

The input arguments contain the estimates and covariance matrix of target parameter, number of target parameters, sample size, and the restriction matrix for each hypothesis under consideration. The output of Bain are the Bayes factor and PMP for each hypothesis. We will use the example from Section 6.2 to illustrate the use of Bain.

The estimates and covariance matrix of target parameters can be obtained using $\mathrm{R}$ package lavaan as shown in Appendix A. For example, from the output of lavaan for the regression model, we observe in $\mathrm{R}$ syntax :

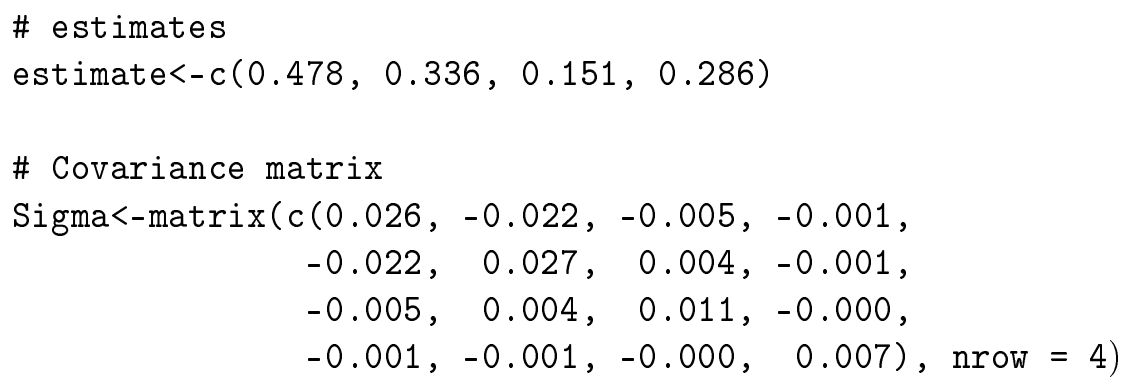

In addition, the sample size is $\mathrm{n}=98$, and the number of target parameters is jointpara $=4$. Argument grouppara indicates the number of group specific parameters, which is zero because in the regression model there is no group specific parameter. Furthermore, argument IRr specifies order constrained hypotheses, while argument ERr for equality constraints will not be used since this paper only deals with order constrained hypotheses. The paragraph below demonstrates how IRr can be constructed to represent order constrained hypotheses.

As was shown in Section 2.2, an order constrained hypothesis $H_{i}$ can be formulated by $\boldsymbol{R}_{i} \theta>\boldsymbol{r}_{i}$. Each constraint $\boldsymbol{R}_{i k} \boldsymbol{\theta}>r_{i k}$ for $k=1, \ldots, K$ in the hypothesis can be written as $R_{i k 1} \theta_{1}+\ldots+R_{i k J} \theta_{J}>r_{i k}$, where $K$ and $J$ are numbers of constraints and parameters in $H_{i}$, respectively. Note that every parameter should be moved to the left hand side of the inequality sign ">", and the constant should be moved to the right hand. In the restriction matrix IRr, the constraint $\boldsymbol{R}_{i k} \boldsymbol{\theta}>r_{i k}$ can be expressed by the line

$$
R_{i k 1} R_{i k 2} \ldots R_{i k J} \quad r_{i k} \text {. }
$$

For example, 
- $\theta_{1}+\theta_{2}+\theta_{3}>0$ corresponds to 1110

- $\theta_{1}-2 \theta_{2}+3 \theta_{3}>0.5$ corresponds to $1-23 \quad 0.5$

- $\theta_{1}-2>\theta_{2}-\theta_{3}$ corresponds to $1-112$

- $\theta_{1}>\theta_{2}>\theta_{3}$ corresponds to $1-1 \quad 000$

$\begin{array}{llll}0 & 1 & -1 & 0\end{array}$

- $\theta_{1}-\theta_{2}>\theta_{3}-\theta_{4}>\theta_{5}-\theta_{6}$ corresponds to

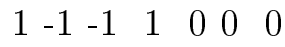

$\begin{array}{llllllllllllll}0 & 0 & 1 & -1 & -1 & 1 & 0\end{array}$

Thus, in the regression model in Section 6.2, three competing order constrained hypotheses $H_{3}: \theta_{1}>\theta_{2}>\theta_{4}>\theta_{1}, H_{4}: \theta_{1}>\theta_{2}>\theta_{3}>\theta_{4}$, and $H_{5}: \theta_{4}>\theta_{3}>\theta_{2}>\theta_{1}$ can be represented in $\mathrm{R}$ script, respectively, by

\# order constrained hypotheses

$\operatorname{IRr} 1<-\operatorname{matrix}(\mathrm{c}(1,-1,0,0,0$,

$0,1,0,-1,0$,

$0,0,-1,1,0)$, nrow $=3$, byrow $=$ TRUE)

IRr2<-matrix $(c(1,-1,0,0,0$,

$0,1,-1,0,0$,

$0,0,1,-1,0)$, nrow $=3$, byrow $=$ TRUE)

$\operatorname{IRr} 3<-\operatorname{matrix}(\mathrm{c}(-1,1,0,0,0$, $0,-1,1,0,0$,

$0,0,-1,1,0)$, nrow $=3$, byrow $=$ TRUE)

\# no equality constrained hypotheses

ERr1<-ERr2<-ERr3<-NULL

Once the estimates and covariance matrix of parameters, number of parameters, sample size, and order constrained hypotheses are specified, running the following line in $\mathrm{R}$ renders test results for the example of regression model: 
res<-Bain (estimate, Sigma, grouppara $=0$, jointpara $=4, \mathrm{n}=98$, ERr1, IRr1, ERr2, IRr2, ERr3, IRr3)

The output of Bain function is stored in a list which consists of \$testResult and \$BFmatrix. The \$testResult reports fits, complexities, Bayes factors and PMPs of each hypothesis under consideration. The \$BFmatrix reports Bayes factor matrix for competing hypotheses from which users can easily obtain Bayes factor $B F_{i i^{\prime}}$ for one hypothesis against another. Take again the regression model for example, the output are

\begin{tabular}{|c|c|c|c|c|c|c|}
\hline & fit & comple & xity & $\mathrm{BF}$ & $\mathrm{PMPa}$ & $\mathrm{PMPb}$ \\
\hline H1 & 0.217 & \multicolumn{2}{|c|}{0.023} & 11.902 & 0.786 & 0.726 \\
\hline $\mathrm{H} 2$ & 0.049 & \multicolumn{2}{|c|}{0.019} & 2.676 & 0.214 & 0.197 \\
\hline H3 & 0.000 & \multicolumn{2}{|c|}{0.019} & 0.010 & 0.001 & 0.001 \\
\hline \multicolumn{7}{|c|}{ round (res\$BFmatrix, 3) } \\
\hline & $\mathrm{H} 1$ & \multicolumn{2}{|c|}{$\mathrm{H} 2$} & \multicolumn{2}{|l|}{ H3 } & \\
\hline H1 & 1.000 & 3.677 & \multicolumn{2}{|c|}{914.137} & & \\
\hline $\mathrm{H} 2$ & 0.272 & 1.000 & \multicolumn{2}{|c|}{248.614} & & \\
\hline H3 & 0.001 & 0.004 & \multicolumn{2}{|c|}{1.000} & & \\
\hline
\end{tabular}

Note that in the first table BF displays Bayes factors of order constrained hypotheses against their complements. In addition, PMPa lists PMPs excluding the unconstrained hypothesis, whereas PMPb includes. In the second table, we can observe for example the Bayes factor for $H_{1}$ against $H_{2}$ is $B F_{12}=3.677$, and the Bayes factor for $H_{2}$ against $H_{1}$ is $B F_{21}=0.272$.

\section{Reference}

Akaike, H. (1973). Information theory and an extension of the maximum likelihood principle. In Proc. 2nd Int. Symp. Information Theory, Ed. B. N. Petrov and F. Csáki. pp.267-81. Budapest: Akademiai kiado.

Barlow, R. E., Bartholomew, D. J., Bremner, J. M., and Brunk, H. D. (1972). Statistical Inference Under Order Restrictions: Theory and Application of Isotonic Regression. New York, NY: John Wiley and Sons.

Bollen, K. A. (1989). Structural Equations with Latent Variables. New York, NY: John Wiley and Sons. 
Burnham, K. P. and Anderson, D. R. (2002). Model Selection and Multimodel Inference: A Practical Information-Theoretic Approach. Second Edition, New York, NY: Springer-Verlag.

Cohen, J. (1994). The earth is round, $\mathrm{p}<.05$. American Psychologist, 49, 997-1003.

DiCiccio, T. J., Kass R. E., Raftery, A., and Wasserman L. (1997). Computing Bayes factors by combining simulation and asymptotic approximations. Journal of the American Statistical Association,92, 903-915.

Gelfand, A. E., Smith, A. F. M., and Lee, T. (1992). Bayesian analysis of constrained parameter and truncated data problems using Gibbs sampling. Journal of the American Statistical Association, 87, 523-532.

Gelman, A., Carlin, J.B., Stern, H.S., and Rubin, D.B. (2004). Bayesian Data Analysis. Second Edition. Boca Raton, FL: Chapman and Hall/CRC.

Gourieroux, C., Holly, A., and Monfort, A. (1982). Likelihood ratio test, Wald test, and Kuhn-Tucker test in linear models with inequality constraints on the regression parameters. Econometrica, 50, 63-80.

Gu, X., Mulder, J., Deković, M., and Hoijtink, H. (2014). Bayesian evaluation of inequality constrained hypotheses. Psychological Methods, 19(4), 511-527.

Hoijtink, H. (2012). Informative Hypotheses: Theory and practice for behavioral and social scientists. Boca Raton, FL: Chapman and Hall/CRC.

Jeffreys, H. (1961). Theory of Probability. Third Edition. Oxford: Oxford University Press.

Jöreskog, K. and Sörbom, D (1979). Advances in factor analysis and structural equation models. Cambridge, Mass: Abt books.

Kass, R.E. and Raftery, A.E. (1995). Bayes factors. Journal of the American Statistical Association, 90, 773-795.

Klugkist, I. and Hoijtink, H. (2007). The Bayes factor for inequality and 
about equality constrained models. Computational Statistics and Data Analysis, 51, 6367-6379.

Klugkist, I., Laudy, O., and Hoijtink, H. (2005). Inequality constrained analysis of variance: A Baysian approach. Psychological Methods, 10, 477-493.

Klugkist, I., Laudy, O., and Hoijtink, H. (2010). Bayesian evaluation of inequality and equality constrained hypotheses for contingency tables. Psychological Methods, 15, 281-299.

Kuiper, R. M. and Hoijtink, H. (2010). Comparisons of means using exploratory and confirmatory approaches. Psychological Methods, 15, 69-86.

Kuiper, R. M., Klugkist, I., and Hoijtink, H. (2010). A Fortran 90 program for confirmatory analysis of variance. Journal of Statistical Software, 34 (8), $1-31$.

Lindley, D. (1957). A statistical paradox. Biometrika, 44, 187-192.

Morey, R. D., Rouder, J. N., Pratte, M. S. and Speckman, P. L. (2011). Using MCMC chain outputs to efficiently estimate Bayes factors. Journal of Mathematical Psychology, 55, 368-378.

Mulder, J. (2014a). Bayes factors for testing inequality constrained hypotheses: Issues with prior specification. British Journal of Mathematical and Statistical Psychology, 67, 153-171.

Mulder, J. (2014b). Prior adjusted default Bayes factors for testing inequality constrained hypotheses. Computational Statistics and Data Analysis, 71, 448-463.

Mulder, J. (2016). Bayes factors for testing order-constrained hypotheses on correlations. Journal of Mathematical Psychology, 72, 104-115.

Mulder, J., Hoijtink, H., and de Leeuw, C. (2012). BIEMS: A Fortran 90 program for calculating Bayes factors for inequality and equality constrained models. Journal of Statistical Software, 46(2), 1-39.

Mulder, J., Hoijtink, H., and Klugkist, I. (2010). Equality and inequality constrained multivariate linear models: objective model selection using con- 
strained posterior priors. Journal of Statistical Planning and Inference, 140, $887-906$.

O'Hagan, A. (1995). Fractional Bayes factors for model comparison. Journal of the Royal Statistical Society. Series B, 57, 99-138.

Rosseel, Y. (2012). lavaan: an R package for structural equation modeling. Journal of Statistical Software, 48(2), 1-36.

Royall, R. (1997). Statistical Evidence: A Likelihood Paradigm. New York, NY: Chapman and Hall/CRC.

Schwarz, G. (1978). Estimating the dimension of a model. Annuals of Statistics, 6(2), 461-464.

Silvapulle, M.J., and Sen, P.K. (2005). Constrained Statistical Inference; Order, Inequality, and Shape Constraints. New York, NY: Wiley.

van de Schoot, R., Hoijtink, H., and Deković, M. (2010). Testing inequality constrained hypotheses in SEM models. Structural Equation Modeling, 17, 443-463.

van de Schoot, R., Hoijtink, H., Hallquist, M.N., and Boelen, P.A. (2012). Bayesian evaluation of inequality constrained hypotheses in SEM models using Mplus. Structural Equation Modeling, 19, 1-17.

Wagenmakers, E. J. (2007). A practical solution to the pervasive problems of $p$ values. Psychonomic Bulletin \& Review, 14, 779-804.

Warren, R. D., White, J. K., and Fuller, W. A. (1974). An errors-in-variables analysis of managerial role performance. Journal of the American Statistical Association, 69, 886-893. 